\title{
LINEAR DIFFERENCE EQUATIONS, FRIEZE PATTERNS, AND THE COMBINATORIAL GALE TRANSFORM
}

\author{
SOPHIE MORIER-GENOUD ${ }^{1}$, VALENTIN OVSIENKO ${ }^{2}$, RICHARD EVAN \\ SCHWARTZ $^{3}$ and SERGE TABACHNIKOV ${ }^{4,5}$ \\ ${ }^{1}$ Sorbonne Universités, UPMC Univ Paris 06, UMR 7586, Institut de Mathématiques de \\ Jussieu-Paris Rive Gauche, Case 247, 4 place Jussieu, F-75005, Paris, France \\ ${ }^{2}$ CNRS, Laboratoire de Mathématiques, U.F.R. Sciences Exactes et Naturelles, Moulin de la \\ Housse - BP 1039, 51687 REIMS Cedex 2, France \\ ${ }^{3}$ Department of Mathematics, Brown University, Providence, RI 02912, USA \\ ${ }^{4}$ Pennsylvania State University, Department of Mathematics, University Park, PA 16802, USA \\ ${ }^{5}$ ICERM, Brown University, Box 1995, Providence, RI 02912, USA; \\ email: tabachni@math.psu.edu
}

Received 20 September 2013; accepted 7 July 2014

\begin{abstract}
We study the space of linear difference equations with periodic coefficients and (anti)periodic solutions. We show that this space is isomorphic to the space of tame frieze patterns and closely related to the moduli space of configurations of points in the projective space. We define the notion of a combinatorial Gale transform, which is a duality between periodic difference equations of different orders. We describe periodic rational maps generalizing the classical Gauss map.
\end{abstract}

2010 Mathematics Subject Classification: 39A70, 14M15, 13F60, 52B99

\section{Introduction}

Linear difference equations appear in many fields of mathematics; they relate fundamental objects of geometry, algebra, and analysis. In this paper, we study the space of linear difference equations with periodic coefficients and (anti)periodic solutions. (The solutions of the equation are periodic if the order of the equation is odd, and antiperiodic if the order is even.) The space of such equations is a

(C) The Author(s) 2014. This is an Open Access article, distributed under the terms of the Creative Commons Attribution licence (http://creativecommons.org/licenses/by/3.0/), which permits unrestricted re-use, distribution, and reproduction in any medium, provided the original work is properly cited. 
very interesting algebraic variety. Despite the fact that this subject is very old and classical, this space has not been studied in detail.

We prove that the space $\mathcal{E}_{k+1, n}$ of $n$-periodic linear difference equations of order $k+1$ is equivalent to the space $\mathcal{F}_{k+1, n}$ of tame $\mathrm{SL}_{k+1}$-frieze patterns of width $w=$ $n-k-2$. (Tameness is the nonvanishing condition on certain determinants; see Definition 2.2.1.) We also show that these spaces are closely related to a certain moduli space $\mathcal{C}_{k+1, n}$ of $n$-gons in $k$-dimensional projective space. While $\mathcal{C}_{k+1, n}$ can be viewed as the quotient of the Grassmannian $\mathrm{Gr}_{k+1, n}$ by a torus action, see [21], the space $\mathcal{E}_{k+1, n}$ is a subvariety of $\mathrm{Gr}_{k+1, n}$. We show that, in the case where $k+1$ and $n$ (and thus $w+1$ and $n$ ) are coprime, the two spaces are isomorphic.

A frieze pattern is just another, more combinatorial, way to represent a linear difference equation with (anti)periodic solutions. However, the theory of frieze patterns was created [8, 9] and developed independently; see [2, 4, 31, 32, 38]. The current interest in this subject is due to the relation with the theory of cluster algebras [5]. We show that the isomorphism between $\mathcal{E}_{k+1, n}$ and $\mathcal{F}_{k+1, n}$ immediately implies the periodicity statement, which is an important part of the theory. Let us mention that a more general notion of $\mathrm{SL}_{k+1}$-tiling was studied in [4]; a version of $\mathrm{SL}_{3}$-frieze patterns, called 2-frieze patterns, was studied in $[32,38]$.

Let us also mention that an $\mathrm{SL}_{k+1}$-frieze pattern can be included in a larger pattern sometimes called a $T$-system (see [11] and references therein), and better known under the name of discrete Hirota equation (see, for a survey, [46]). Although an $\mathrm{SL}_{k+1}$-frieze pattern is a small part of a solution of a $T$-system, it contains all the information about the solution. We will not use this viewpoint in the present paper.

The main result of this paper is a description of the duality between the spaces $\mathcal{E}_{k+1, n}$ and $\mathcal{E}_{w+1, n}$ when $(k+1)+(w+1)=n$. We call this duality the combinatorial Gale transform. This is an analog of the classical Gale transform, which is a well-known duality on the moduli spaces of point configurations; see $[6,7, \mathbf{1 4}, \mathbf{1 7}]$. We think that the most interesting feature of the combinatorial Gale transform is that it allows one to change the order of an equation while keeping all the information about it.

Let us give here the simplest example, which is related to Gauss' pentagramma mirificum [18]. Consider a third-order difference equation

$$
V_{i}=a_{i} V_{i-1}-b_{i} V_{i-2}+V_{i-3}, \quad i \in \mathbb{Z} .
$$

Assume that the coefficients $\left(a_{i}\right)$ and $\left(b_{i}\right)$ are 5-periodic $\left(a_{i+5}=a_{i}\right.$ and $b_{i+5}=$ $b_{i}$ ), and that all the solutions $\left(V_{i}\right)$ are also 5-periodic, that is, $V_{i+5}=V_{i}$. The combinatorial Gale transform in this case consists in 'forgetting the coefficients $b_{i}{ }^{\prime}$; to this equation it associates the difference equation of order 2 : 


$$
W_{i}=a_{i} W_{i-1}-W_{i-2} .
$$

It turns out that the solutions of the latter equation are 5-antiperiodic: $W_{i+5}=$ $-W_{i}$. Conversely, as we will explain later, one can reconstruct the initial thirdorder equation from the second-order one. Geometrically speaking, this transform sends (projective equivalence classes of) pentagons in $\mathbb{P}^{2}$ to those in $\mathbb{P}^{1}$. In terms of the frieze patterns, this corresponds to a duality between 5-periodic Coxeter friezes and 5-periodic $\mathrm{SL}_{3}$-friezes.

Our study is motivated by recent works $[19,20,25,26,29,30,34,35,42,43]$ on a certain class of discrete integrable systems arising in projective differential geometry and cluster algebra. The best known among these maps is the pentagram map $[40,41]$ acting on the moduli space of $n$-gons in the projective plane.

We would like to attract attention to a recent paper by Krichever [27] that connects the combinatorial Gale transform and the classical theory of commuting difference operators. This provides a link with the theory of completely integrable systems; see [28].

This paper is organized as follows.

In Section 2, we introduce the main objects, namely the spaces $\mathcal{E}_{k+1, n}, \mathcal{F}_{k+1, n}$, and $\mathcal{C}_{k+1, n}$.

In Section 3, we construct an embedding of $\mathcal{E}_{k+1, n}$ into the Grassmannian $\mathrm{Gr}_{k+1, n}$. We then formulate the result about the isomorphism between $\mathcal{E}_{k+1, n}$ and $\mathcal{F}_{k+1, n}$, and give an explicit construction of this isomorphism. We also define a natural map from the space of equations to that of configurations of points in the projective space.

In Section 4, we introduce the Gale transform, which is the main notion of this paper. We show that a difference equation corresponds not to just one, but to two different frieze patterns. This is what we call the Gale duality. We also introduce a more elementary notion of projective duality that commutes with the Gale transform.

In Section 5, we calculate explicitly the entries of the frieze pattern associated with a difference equation. We give explicit formulas for the Gale transform. These formulas are similar to the classical and well-known expressions often called the André determinants; see [1, 22].

Relation of the Gale transform to representation theory is described in Section 6. We represent an $\mathrm{SL}_{k+1}$-frieze pattern (and thus a difference equation) in a form of a unitriangular matrix. We prove that the Gale transform coincides with the restriction of the involution of the nilpotent group of unitriangular matrices introduced and studied by Berenstein et al. [3].

In Section 7, we present an application of the isomorphism between difference equations and frieze patterns. We construct rational periodic maps generalizing the Gauss map. These maps are obtained by calculating consecutive coefficients 
of (anti)periodic second-order and third-order difference equations and using the periodicity property of $\mathrm{SL}_{2}$-frieze and $\mathrm{SL}_{3}$-frieze patterns. We also explain how these rational maps can be derived, in an alternate way, from the projective geometry of polygons.

In Section 8, we explain the details about the relations between the spaces we consider. We also outline relations to the Teichmüller theory.

\section{Difference equations, $\mathrm{SL}_{k+1}$-frieze patterns, and polygons in $\mathbb{R P}^{k}$}

In this section, we will define the three closely related spaces $\mathcal{E}_{k+1, n}, \mathcal{F}_{k+1, n}$, and $\mathcal{C}_{k+1, n}$ discussed in the introduction. All three spaces will be equipped with the structure of algebraic variety; we choose $\mathbb{R}$ as the ground field.

2.1. Difference equations. Let $\mathcal{E}_{k+1, n}$ be the space of order $k+1$ difference equations

$$
V_{i}=a_{i}^{1} V_{i-1}-a_{i}^{2} V_{i-2}+\cdots+(-1)^{k-1} a_{i}^{k} V_{i-k}+(-1)^{k} V_{i-k-1},
$$

where $a_{i}^{j} \in \mathbb{R}$, with $i \in \mathbb{Z}$ and $1 \leqslant j \leqslant k$, are coefficients and $V_{i}$ are unknowns. (Note that the superscript $j$ is an index, not a power.) Throughout the paper, a solution $\left(V_{i}\right)$ will consist either of real numbers, $V_{i} \in \mathbb{R}$, or of real vectors $V_{i} \in$ $\mathbb{R}^{k+1}$. We always assume that the coefficients are periodic with some period $n \geqslant$ $k+2$,

$$
a_{i+n}^{j}=a_{i}^{j}
$$

for all $i, j$, and that all the solutions are $n$-(anti)periodic:

$$
V_{i+n}=(-1)^{k} V_{i}
$$

The algebraic variety structure on $\mathcal{E}_{k+1, n}$ will be introduced in Section 3.1.

EXAMPLE 2.1.1. The simplest example of a difference equation (2.1), an element of the space $\mathcal{E}_{2, n}$, is the well-known discrete Hill (or Sturm-Liouville) equation

$$
V_{i}=a_{i} V_{i-1}-V_{i-2}
$$

with $n$-periodic coefficients and $n$-antiperiodic solutions.

2.2. $\mathbf{S L}_{k+1}$-frieze patterns. An $\mathrm{SL}_{k+1}$-frieze pattern (see [4]) is an array of numbers consisting of a finite number of infinite rows: 
0

$\cdots$

. $\quad d_{0,1}$

$d_{0,0}$

.. 1

0

1

1

0
0

.. $\quad d_{0, w-1}$

1

0

1

$d_{1, w}$

$d_{2, w+1}$

$\therefore$

$d_{1,2}$ $d_{1,1}$

0
0

:

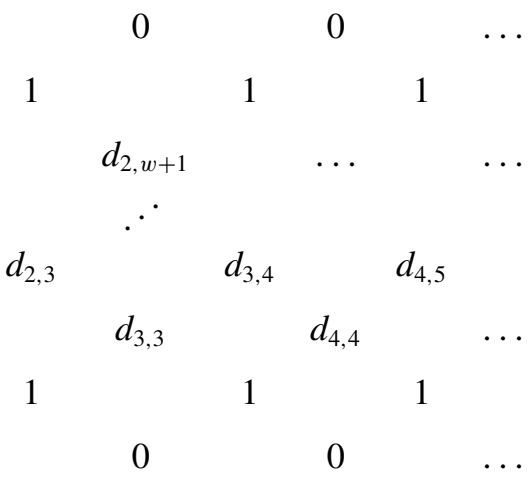

(2.3)

where the strip is bounded by $k$ rows of 0 s at the top, and at the bottom, and where every 'diamond' $(k+1) \times(k+1)$-subarray forms an element of $\mathrm{SL}_{k+1}$. The number of rows between the bounding rows of $1 \mathrm{~s}$ is called the width, and is denoted by $w$. To simplify the pictures, we often omit the bounding rows of 0 s.

More precisely, the entries of the frieze are denoted by $\left(d_{i, j}\right)$, where $i \in \mathbb{Z}$, and

$$
i-k-1 \leqslant j \leqslant i+w+k,
$$

for a fixed $i$, and they satisfy the following 'boundary conditions',

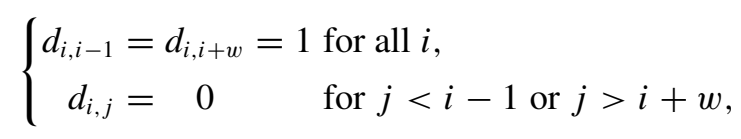

and the ' $\mathrm{SL}_{k+1}$-conditions'

$$
D_{i, j}:=\left|\begin{array}{llll}
d_{i, j} & d_{i, j+1} & \ldots & d_{i, j+k} \\
d_{i+1, j} & d_{i+1, j+1} & \ldots & d_{i+1, j+k} \\
\ldots & \ldots & & \ldots \\
d_{i+k, j} & d_{i+k, j+1} & \ldots & d_{i+k, j+k}
\end{array}\right|=1,
$$

for all $(i, j)$ in the index set.

DEFINITION 2.2.1. An $\mathrm{SL}_{k+1}$-frieze pattern is called tame if every $(k+2) \times(k+$ 2)-determinant equals 0 .

The notion of tame friezes was introduced in [4]. Let $\mathcal{F}_{k+1, n}$ denote the space of tame $\mathrm{SL}_{k+1}$-frieze patterns of width $w=n-k-2$. 
EXAMPLE 2.2.2. (a) The most classical example of friezes is that of CoxeterConway frieze patterns $[8,9]$ corresponding to $k=1$. For instance, a generic Coxeter-Conway frieze pattern of width 2 looks like

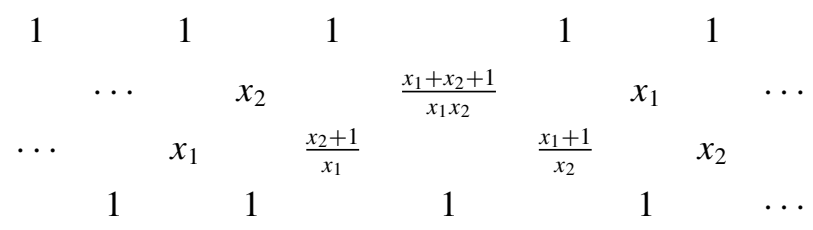

for some $x_{1}, x_{2}$. (Note that we omitted the first and the last rows of $0 \mathrm{~s}$.) This example is related to so-called Gauss pentagramma mirificum [18]; see also [39].

(b) The following width-3 Coxeter pattern is not tame:

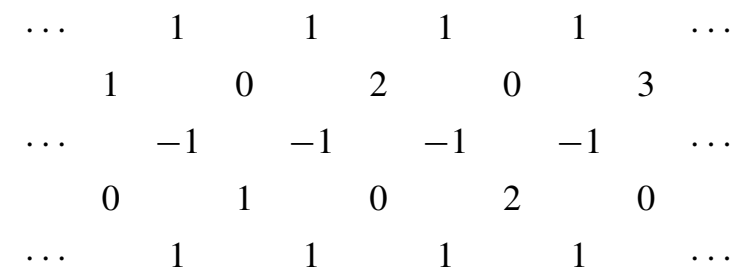

REMARK 2.2.3. Generic $\mathrm{SL}_{k+1}$-frieze patterns are tame. We understand the genericity of an $\mathrm{SL}_{k+1}$-frieze pattern as the condition that every $k \times k$-determinant is different from 0 . Then the vanishing of the $(k+2) \times(k+2)$-determinants follows from the Dodgson condensation formula, involving minors of order $k+2, k+1$, and $k$ obtained by erasing the first and/or last row/column. The formula can be pictured as follows:

$$
\left|\begin{array}{cccc}
* & * & * & * \\
* & * & * & * \\
* & * & * & * \\
* & * & * & *
\end{array}\right|\left|\begin{array}{ll}
* & * \\
* & *
\end{array}\right|=\left|\begin{array}{ccc}
* & * & * \\
* & * & * \\
* & * & *
\end{array}\right|\left|\begin{array}{ccc}
* & * & * \\
* & * & * \\
* & * & *
\end{array}\right|-\left|\begin{array}{ccc}
* & * & * \\
* & * & * \\
* & * & * \\
& &
\end{array}\right|\left|\begin{array}{lll}
* & * & * \\
* & * & * \\
* & * & *
\end{array}\right|
$$

where the deleted columns/rows are left blank.

Notation 2.2.4. Given an $\mathrm{SL}_{k+1}$-frieze pattern $F$ as in (2.3), it will be useful to define the following $(k+1) \times n$-matrices

$$
M_{F}^{(i)}:=\left(\begin{array}{cccccccc}
1 & d_{i, i} & \ldots & \ldots & d_{i, w+i-1} & 1 & & \\
& \ddots & & & & & \ddots & \\
& & & & & & & \\
& 1 & d_{k+i, k+i} & \ldots & \ldots & d_{k+i, k+w+i-1} & 1
\end{array}\right) .
$$


Throughout this paper, the 'empty' entries of matrices represent 0 . For a subset $I$ of $k+1$ consecutive elements of $\mathbb{Z} / n \mathbb{Z}$, denote by $\Delta_{I}(M)$ the minor of a matrix $M$ based on columns with indices in $I$. There are $n$ such intervals $I$. By the definition of an $\mathrm{SL}_{k+1}$-frieze pattern, $\Delta_{I}\left(M_{F}^{(i)}\right)=1$.

The matrix $M_{F}^{(i)}$ determines a unique tame $\mathrm{SL}_{k+1}$-frieze pattern. Indeed, from $M_{F}^{(i)}$ one can compute all the entries $d_{i, j}$, one after another, using the fact that $(k+2) \times(k+2)$-minors of the frieze pattern vanish.

We will also denote the North-East (respectively South-East) diagonals of an $\mathrm{SL}_{k+1}$-frieze pattern by $\mu_{i}$ (respectively $\eta_{j}$ ):

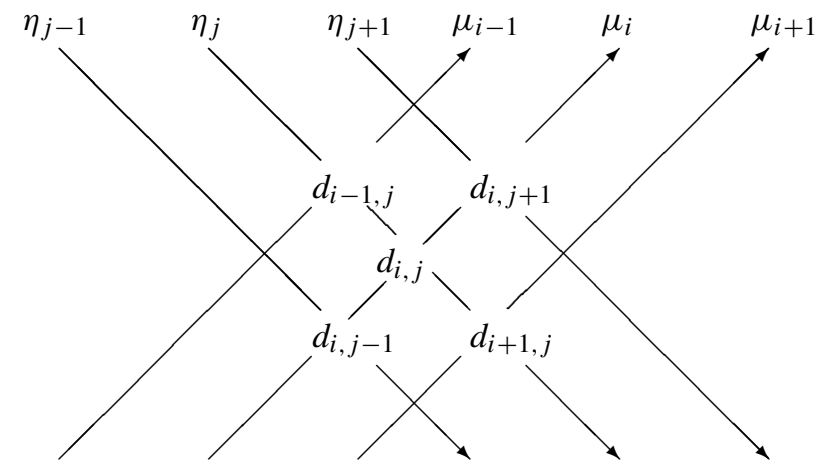

2.3. Moduli space of polygons. A nondegenerate n-gon is a map

$$
v: \mathbb{Z} \rightarrow \mathbb{R P}^{k}
$$

such that $v_{i+n}=v_{i}$, for all $i$, and no $k+1$ consecutive vertices belong to the same hyperplane.

Let $\mathcal{C}_{k+1, n}$ be the moduli space of projective equivalence classes of nondegenerate $n$-gons in $\mathbb{R P}^{k}$.

REMARK 2.3.1. The space $\mathcal{C}_{k+1, n}$ has been extensively studied; see, for example, $[14,17,21,23]$. Our interest in this space is motivated by the study of the pentagram map, a completely integrable map on the space $\mathcal{C}_{3, n}$ (see $[34,35$, 40, 41, 43]), as well as its higher generalizations acting on $\mathcal{C}_{k, n}$, for $k \geqslant 4$ (see $[25,26,29,30])$. Let us add that the Gale transform interchanges the spaces $\mathcal{C}_{k, n}$ and $\mathcal{C}_{n-k, n}$, and it is interesting to investigate how it interacts with various discrete dynamical systems on these spaces.

\section{Geometric description of the spaces $\mathcal{E}_{k+1, n}, \mathcal{F}_{k+1, n}$, and $\mathcal{C}_{k+1, n}$}

In this section, we describe the structures of algebraic varieties on the spaces $\mathcal{E}_{k+1, n}, \mathcal{F}_{k+1, n}$, and $\mathcal{C}_{k+1, n}$. We also prove that the spaces $\mathcal{E}_{k+1, n}$ and $\mathcal{F}_{k+1, n}$ are 
isomorphic. The spaces $\mathcal{E}_{k+1, n}$ and $\mathcal{C}_{k+1, n}$ are also closely related. We will define a map from $\mathcal{E}_{k+1, n}$ to $\mathcal{C}_{k+1, n}$, which turns out to be an isomorphism, provided that $k+1$ and $n$ are coprime. If this is not the case, then these two spaces are different.

3.1. The structure of an algebraic variety on $\mathcal{E}_{k+1, n}$. The space of difference equations $\mathcal{E}_{k+1, n}$ is an affine algebraic subvariety of $\mathbb{R}^{n k}$. This structure is defined by condition (2.2).

The space of solutions of Equation $(2.1)$ is $(k+1)$-dimensional. Consider $k+$ 1 linearly independent solutions forming a sequence $\left(V_{i}\right)_{i \in \mathbb{Z}}$ of vectors in $\mathbb{R}^{k+1}$ satisfying (2.1). Since the coefficients are $n$-periodic, there exists a linear map $T$ on the space of solutions called the monodromy satisfying

$$
V_{i+n}=T V_{i}
$$

One can view the monodromy as an element of $\mathrm{GL}_{k+1}$ defined up to a conjugation.

Proposition 3.1.1. The space $\mathcal{E}_{k+1, n}$ has codimension $k(k+2)$ in $\mathbb{R}^{n k}$ with coordinates $a_{i}^{j}$.

Proof. Since the last coefficient in (2.1) is $(-1)^{k}$, one has (Throughout the paper, $\left|V_{i}, \ldots, V_{i+k}\right|$ stands for the determinant of the matrix with columns $V_{i}, \ldots$, $V_{i+k}$.)

$$
\left|V_{i}, V_{i+1}, \ldots, V_{i+k}\right|=\left|V_{i+1}, V_{i+2}, \ldots, V_{i+k+1}\right| .
$$

Hence the monodromy is volume preserving, and thus belongs to the group $\mathrm{SL}_{k+1}(\mathbb{R})$.

If furthermore all the solutions of (2.1) are $n$-(anti)periodic, then the monodromy is $(-1)^{k} \mathrm{Id}$. Since $\operatorname{dim} \mathrm{SL}_{k+1}=k(k+2)$, this gives $k(k+2)$ polynomial relations on the coefficients.

\subsection{Embedding of $\mathcal{F}_{k+1, n}$ into the Grassmannian. Consider the} Grassmannian $\mathrm{Gr}_{k+1, n}$ of $(k+1)$-dimensional subspaces in $\mathbb{R}^{n}$. Let us show that the space of $\mathrm{SL}_{k+1}$-frieze patterns $\mathcal{F}_{k+1, n}$ can be viewed as an $(n-1)$ codimensional algebraic subvariety of $\mathrm{Gr}_{k+1, n}$.

Recall that the Grassmannian can be described as the quotient

$$
\mathrm{Gr}_{k+1, n} \simeq \mathrm{GL}_{k+1} \backslash \mathrm{Mat}_{k+1, n}^{*}(\mathbb{R}),
$$

where $\operatorname{Mat}_{k+1, n}^{*}(\mathbb{R})$ is the set of real $(k+1) \times n$-matrices of rank $k+1$. Every point of $\mathrm{Gr}_{k+1, n}$ can be represented by a $(k+1) \times n$-matrix $M$. The Plücker coordinates on $\mathrm{Gr}_{k+1, n}$ are all the $(k+1) \times(k+1)$-minors of $M$; see, for example [16]. 
Note that the $(k+1) \times(k+1)$-minors depend on the choice of the matrix, but they are defined up to a common factor. Therefore, the Plücker coordinates are homogeneous coordinates independent of the choice of the representing matrix. The minors $\Delta_{I}(M)$ with consecutive columns, see Notation 2.2.4, play a special role.

PROPOSITION 3.2.1. The subvariety of $G r_{k+1, n}$ consisting in the elements that can be represented by the matrices such that all the minors $\Delta_{I}(M)$ are equal to each other,

$$
\Delta_{I}(M)=\Delta_{I^{\prime}}(M) \text { for all } I, I^{\prime},
$$

is in one-to-one correspondence with the space $\mathcal{F}_{k+1, n}$ of $\mathrm{SL}_{k+1}$-frieze patterns.

Proof. If $M \in \mathrm{Gr}_{k+1, n}$ satisfies $\Delta_{I}(M)=\Delta_{I^{\prime}}(M)$ for all intervals $I, I^{\prime}$, then it is easy to see that there is a unique representative of $M$ of the form (2.5), and therefore a unique corresponding $\mathrm{SL}_{k+1}$-frieze pattern.

Conversely, given an $\mathrm{SL}_{k+1}$-frieze pattern $F$, the corresponding matrices $M_{F}^{(i)}$ satisfy (3.7). Fixing $i$, for instance, taking $i=1$, we obtain a well-defined embedding

$$
\mathcal{F}_{k+1, n} \subset \mathrm{Gr}_{k+1, n} .
$$

Hence the result follows.

Note that the constructed embedding of the space $\mathcal{F}_{k+1, n}$ into the Grassmannian depends on the choice of index $i$. A different choice leads to a different embedding.

3.3. The space $\mathcal{C}_{k+1, n}$ as a quotient of the Grassmannian. A classical way to describe the space $\mathcal{C}_{k+1, n}$ as the quotient of $\mathrm{Gr}_{k+1, n}$ by the torus action,

$$
\mathcal{C}_{k+1, n} \simeq \mathrm{Gr}_{k+1, n} / \mathbb{T}^{n-1},
$$

is due to Gelfand and MacPherson [21].

Let us comment on this realization of $\mathcal{C}_{k+1, n}$. Given an $n$-gon $v: \mathbb{Z} \rightarrow \mathbb{R P}^{k}$, consider an arbitrary lift $V: \mathbb{Z} \rightarrow \mathbb{R}^{k+1}$. The result of such a lift is a full rank $(k+1) \times n$-matrix, and thus an element of $\mathrm{Gr}_{k+1, n}$. Recall that the action of $\mathbb{T}^{n-1}$, in terms of the matrix realization (3.6), consists in multiplying $(k+1) \times n$-matrices by diagonal $n \times n$-matrices with determinant 1 . The projection of $V$ to the quotient $\mathrm{Gr}_{k+1, n} / \mathbb{T}^{n-1}$ is independent of the lift of $v$ to $\mathbb{R}^{k+1}$.

3.4. Triality. Let us briefly explain the relations between the spaces $\mathcal{E}_{k+1, n}$, $\mathcal{F}_{k+1, n}$, and $\mathcal{C}_{k+1, n}$. We will give more details in Section 8 . 
The spaces of difference equations $\mathcal{E}_{k+1, n}$ and that of $\mathrm{SL}_{k+1}$-frieze patterns $\mathcal{F}_{k+1, n}$ are always isomorphic. These spaces are, in general, different from the moduli space of $n$-gons, but isomorphic to it if $k+1$ and $n$ are coprime.

THEOREM 3.4.1. (i) The spaces $\mathcal{E}_{k+1, n}$ and $\mathcal{F}_{k+1, n}$ are isomorphic algebraic varieties.

(ii) If $k+1$ and $n$ are coprime, then the spaces $\mathcal{E}_{k+1, n}, \mathcal{F}_{k+1, n}$, and $\mathcal{C}_{k+1, n}$ are isomorphic algebraic varieties.

The complete proof of this theorem will be given in Section 8 . Here, we just construct the isomorphisms.

Part (i). Let us define a map

$$
\mathcal{E}_{k+1, n} \stackrel{\simeq}{\longrightarrow} \mathcal{F}_{k+1, n}
$$

which identifies the two spaces. Roughly speaking, we generate the solutions of the recurrence equation (2.1), starting with $k 0 \mathrm{~s}$, followed by 1 , and put them on the North-East diagonals of the frieze pattern. Let us give a more detailed construction.

Given a difference equation (2.1) satisfying the (anti)periodicity assumption (2.2), we define the corresponding $\mathrm{SL}_{k+1}$-frieze pattern (2.3) by constructing its North-East diagonals $\mu_{i}$. This diagonal is given by a sequence of real numbers $V=\left(V_{s}\right)_{s \in \mathbb{Z}}$ that are the solution of Equation (2.1) with the initial condition

$$
\left(V_{i-k-1}, V_{i-k}, \ldots, V_{i-1}\right)=(0,0, \ldots, 0,1)
$$

this defines the numbers $d_{i, j}$ via

$$
d_{i, j}:=V_{j}
$$

Since the solution is $n$-(anti)periodic, we have

$$
d_{i, i+w+1}=\cdots=d_{i, i+n-2}=0, \quad d_{i, i+n-1}=(-1)^{k} .
$$

Furthermore, from Equation (2.1), one has

$$
d_{i, i+w}=1 .
$$

The sequence of (infinite) vectors $\left(\eta_{i}\right)$, that is, of South-East diagonals, satisfies Equation (2.1).

EXAMPLE 3.4.2. For an arbitrary difference equation, the first coefficients of the $i$ th diagonal are 


$$
\begin{aligned}
d_{i, i} & =a_{i}^{1}, \\
d_{i, i+1} & =a_{i}^{1} a_{i+1}^{1}-a_{i+1}^{2}, \\
d_{i, i+2} & =a_{i}^{1} a_{i+1}^{1} a_{i+2}^{1}-a_{i+1}^{2} a_{i+2}^{1}-a_{i}^{1} a_{i+2}^{2}+a_{i+2}^{3} .
\end{aligned}
$$

We will give more general determinant formulas in Section 5.

REMARK 3.4.3. The idea that the diagonals of a frieze pattern satisfy a difference equation goes back to Conway and Coxeter [8]. The isomorphism between the spaces of difference equations and frieze patterns was used in $[32,34]$ in the cases $k=1,2$.

Parts (ii). The construction of the map $\mathcal{E}_{k+1, n} \rightarrow \mathcal{C}_{k+1, n}$ consists of the following two steps.

1. The spaces $\mathcal{E}_{k+1, n}$ and $\mathcal{F}_{k+1, n}$ are isomorphic.

2. There is an embedding $\mathcal{F}_{k+1, n} \subset \mathrm{Gr}_{k+1, n}$, and there is a projection $\mathrm{Gr}_{k+1, n} \rightarrow$ $\mathcal{C}_{k+1, n}$; see (2.5) and (3.8).

More directly, given a difference equation (2.1) with (anti)periodic solutions, the space of solutions being $k+1$-dimensional, we choose any linearly independent solutions $\left(V_{i}^{(1)}\right), \ldots,\left(V_{i}^{(k+1)}\right)$. For every $i$, we obtain a point, $V_{i} \in \mathbb{R}^{k+1}$, which we project to $\mathbb{R} \mathbb{P}^{k}$; the (anti)periodicity assumption implies that we obtain an $n$-gon. Furthermore, the constructed $n$-gon is nondegenerate, since the $(k+1) \times(k+1)$-determinant

$$
\left|V_{i}, V_{i+1}, \ldots, V_{i+k}\right|=\text { Const } \neq 0 .
$$

A different choice of solutions leads to a projectively equivalent $n$-gon. We have constructed a map

$$
\mathcal{E}_{k+1, n} \longrightarrow \mathcal{C}_{k+1, n}
$$

We will see, in Section 8.2, that this map is an isomorphism if and only if $k+1$ and $n$ are coprime.

Proposition 3.4.4. Suppose that $\operatorname{gcd}(n, k+1)=q \neq 1$; then the image of the constructed map has codimension $q-1$.

We will give the proof in Section 8.3.

Notation 3.4.5. It will be convenient to write the $\mathrm{SL}_{k+1}$-frieze pattern associated with a difference equation (2.1) in the following form: 


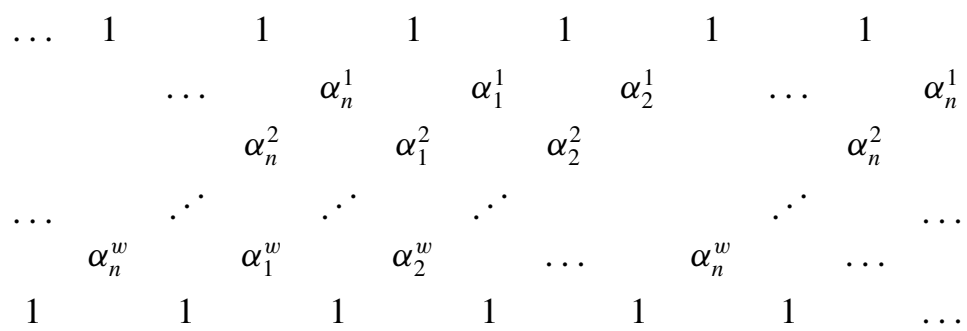

The relation between the old and new notation for the entries of the $\mathrm{SL}_{k+1}$-frieze pattern of width $w$ is

$$
d_{i, j}=\alpha_{i-1}^{w-j+i}, \quad \alpha_{i}^{j}=d_{i+1, w+i-j+1},
$$

where $d_{i, j}$ is the general notation for the entries of an $\mathrm{SL}_{k+1}$-frieze pattern. See formula (2.3).

\section{The combinatorial Gale transform}

In this section, we present another isomorphism between the introduced spaces. This is a combinatorial analog of the classical Gale transform, and it results from the natural isomorphism of Grassmannians:

$$
\mathrm{Gr}_{k+1, n} \simeq \mathrm{Gr}_{w+1, n}
$$

for $n=k+w+2$.

On the spaces $\mathcal{E}_{k+1, n}$ and $\mathcal{F}_{k+1, n}$, we also define an involution, which is a combinatorial version of the projective duality. The Gale transform commutes with the projective duality so both maps define an action of the Klein group $(\mathbb{Z} / 2 \mathbb{Z})^{2}$.

\subsection{Statement of the result.}

DEFINITION 4.1.1. We say that a difference equation (2.1) with $n$-(anti)periodic solutions is the Gale dual of the following difference equation of order $w+1$ :

$$
W_{i}=\alpha_{i}^{1} W_{i-1}-\alpha_{i}^{2} W_{i-2}+\cdots+(-1)^{w-1} \alpha_{i}^{w} W_{i-w}+(-1)^{w} W_{i-w-1},
$$

where $\alpha_{r}^{s}$ are the entries of the $\mathrm{SL}_{k+1}$-frieze pattern (3.12) corresponding to (2.1).

The following statement is the main result of the paper.

THEOREM 4.1.2. (i) All solutions of Equation (4.14) are $n$-(anti)periodic; that is, 


$$
W_{i+n}=(-1)^{w} W_{i} .
$$

(ii) The defined map $\mathcal{E}_{k+1, n} \rightarrow \mathcal{E}_{w+1, n}$ is an involution.

We obtain an isomorphism

$$
\mathcal{G}: \mathcal{E}_{k+1, n} \stackrel{\simeq}{\longrightarrow} \mathcal{E}_{w+1, n}
$$

between the spaces of $n$-(anti)periodic difference equations of orders $k+1$ and $w+1$, provided that

$$
n=k+w+2 \text {. }
$$

We call this isomorphism the combinatorial Gale duality (or the combinatorial Gale transform).

An equivalent way to formulate the above theorem is to say that there is a duality between $\mathrm{SL}_{k+1}$-frieze patterns of width $w$ and $\mathrm{SL}_{w+1}$-frieze patterns of width $k$ :

$$
\mathcal{G}: \mathcal{F}_{k+1, n} \stackrel{\simeq}{\longrightarrow} \mathcal{F}_{w+1, n} .
$$

Proposition 4.1.3. The $\mathrm{SL}_{w+1}$-frieze pattern associated to Equation (4.14) is the following:

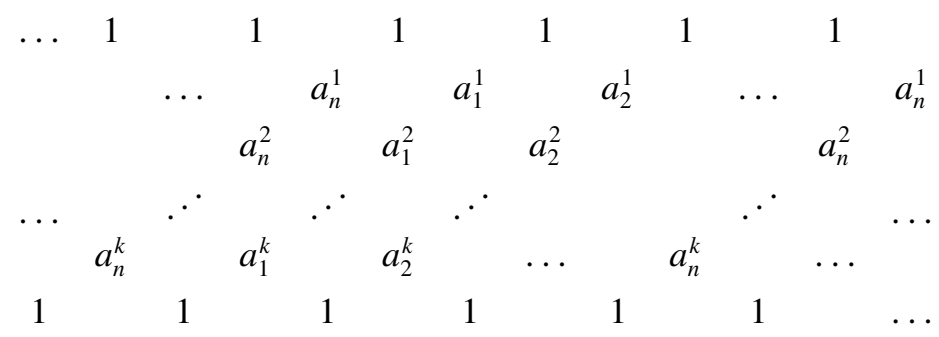

We say that the $\mathrm{SL}_{w+1}$-frieze pattern (4.16) is Gale dual to the $\mathrm{SL}_{k+1}$-frieze pattern (3.12).

The combinatorial Gale transform is illustrated by Figure 1.

4.2. Proof of Theorem 4.1.2 and Proposition 4.1.3. Let us consider the following $n$-(anti)periodic sequence $\left(W_{i}\right)$. On the interval $\left(W_{-w}, \ldots, W_{n-w-1}\right)$ of length $n$, we set

$$
\begin{gathered}
\left(W_{-w}, \ldots, W_{-1}, W_{0}, W_{1}, W_{2}, \ldots, W_{n-w-2}, W_{n-w-1}\right) \\
:=\left(0, \ldots, 0,1, a_{n}^{k}, a_{n}^{k-1}, \ldots, a_{n}^{1}, 1\right)
\end{gathered}
$$

and then continue by (anti)periodicity: $W_{i+n}=(-1)^{w} W_{i}$. 
LEMMA 4.2.1. The constructed sequence $\left(W_{i}\right)$ satisfies Equation (4.14).

Proof of the lemma. By construction of the frieze pattern (3.12), its South-East diagonals $\eta_{i}$ satisfy (2.1).

Consider the following selection of $k+2$ diagonals in the frieze pattern (3.12), and form the following $(k+2) \times n$-matrix:

$$
\left(\begin{array}{ccccc}
\eta_{n-k-1} & \eta_{n-k} & & & \eta_{n} \\
\alpha_{1}^{1} & 1 & 0 & \ldots & 0 \\
\alpha_{2}^{2} & \alpha_{2}^{1} & 1 & 0 & \vdots \\
\vdots & & \ddots & \ddots & 0 \\
\vdots & & & \alpha_{k+1}^{1} & 1 \\
\alpha_{w}^{w} & & & & \alpha_{k+2}^{1} \\
1 & \alpha_{w+1}^{w} & & & \vdots \\
0 & \ddots & & & \vdots \\
\vdots & & 1 & \alpha_{n-2}^{w} & \alpha_{n-2}^{w-1} \\
0 & & 0 & 1 & \alpha_{n-1}^{w} \\
(-1)^{k} & 0 & \cdots & 0 & 1
\end{array}\right) .
$$

The above diagonals satisfy the equation

$$
\eta_{n}=a_{n}^{1} \eta_{n-1}-\cdots-(-1)^{k} a_{n}^{k} \eta_{n-k}+(-1)^{k} \eta_{n-k-1} .
$$

Let us express this equation for each component (that is, each row of the above matrix).

The first row gives $a_{n}^{k}=\alpha_{1}^{1}$, which can be rewritten as $W_{1}=\alpha_{1}^{1} W_{0}$. Since, by construction, $W_{-1}=\cdots=W_{-w}=0$, we can rewrite this relation as

$$
W_{1}=\alpha_{1}^{1} W_{0}-\alpha_{1}^{2} W_{-1}+\cdots+(-1)^{w} W_{-w},
$$

which is precisely (4.14) for $i=1$. Then, considering the second component of the vectors $\eta$, we obtain the relation $0=a_{n}^{k-1}-a_{n}^{k} \alpha_{2}^{1}+\alpha_{2}^{2}$, which reads as

$$
W_{2}=\alpha_{2}^{1} W_{1}-\alpha_{2}^{2} W_{0} \Longleftrightarrow W_{2}=\alpha_{2}^{1} W_{1}-\alpha_{2}^{2} W_{0}+\alpha_{2}^{3} W_{-1}+\cdots+(-1)^{w} W_{1-w} .
$$




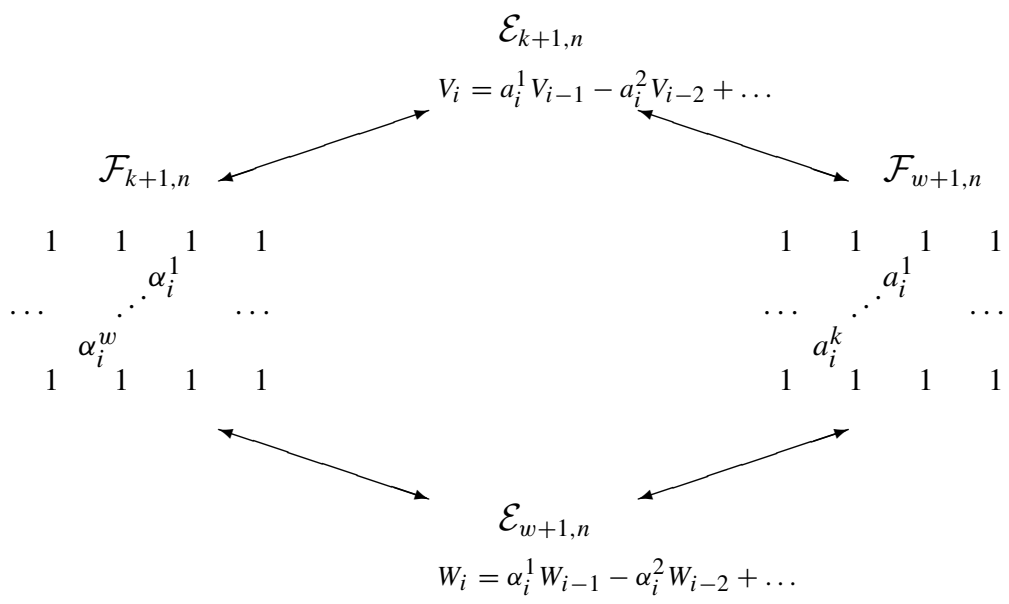

Figure 1. Duality between periodic difference equations, friezes of solutions, and friezes of coefficients; the explicit relations between the coefficients $a_{i}^{j}$ and $\alpha_{i}^{j}$ will be given in Proposition 5.1.1.

Continuing this process, we obtain $n$ relations which correspond precisely to Equation (4.14), for $i=1, \ldots, n$. Hence the lemma follows.

Shifting the indices, in a similar way we obtain $w$ other solutions of Equation (4.14) that, on the period $\left(W_{-w}, \ldots, W_{n-w-1}\right)$, are given by
$(0$
$0, \quad 1, \quad a_{n-1}^{k}$,
$a_{n-1}^{k-1}$,
$a_{n-1}^{1}, \quad 1$,
$(0, \quad \ldots$
$1, \quad a_{n-2}^{k}$,
$a_{n-2}^{k-1}$,
$\ldots, \quad a_{n-2}^{1}$,
$1, \quad 0$,
$\left(1, \quad a_{n-w}^{k}, \quad a_{n-w}^{k-1}, \quad \ldots, \quad a_{n-w}^{1}, \quad 1\right.$,
0
$0, \quad 0)$

Together with the solution from Lemma 4.2.1, these solutions are linearly independent and therefore form a basis of $n$-(anti)periodic solutions of Equation (4.14). We proved that this equation indeed belongs to $\mathcal{E}_{w+1, n}$, so that the map $\mathcal{G}$ is well defined.

The relation between Equations (2.1) and (4.14) can be described as follows: the solutions of the former one are the coefficients of the latter, and vice versa. Therefore, the map $\mathcal{G}$ is an involution. This finishes the proof of Theorem 4.1.2.

To prove Proposition 4.1.3, it suffices to notice that the diagonals of the pattern (4.16) are exactly the solutions of Equation (4.14) with initial conditions 
$\left(0, \ldots, 0,1, a_{i}^{k}\right)$. This is exactly the way we associate a frieze pattern to a differential equation; see Section 3.4.

EXAMPLE 4.2.2. Let us give the most elementary, but perhaps the most striking, example of the combinatorial Gale transform. Suppose that a difference equation (2.1) of order $k+1$ is such that all its solutions $\left(V_{i}\right)$ are $(k+3)$-(anti)periodic:

$$
V_{i+k+3}=(-1)^{k} V_{i}
$$

Consider the Hill equation

$$
W_{i}=a_{i}^{1} W_{i-1}-W_{i-2},
$$

obtained by 'forgetting' the coefficients $a_{i}^{j}$ with $j \geqslant 2$. Theorem 4.1.2 then implies that all the solutions of this equation are antiperiodic with the same period: $W_{i+k+3}=-W_{i}$.

Conversely, any difference equation (2.1) of order $k+1$ with $(k+3)$ (anti)periodic solutions can be constructed out of a Hill equation.

At first glance, it appears paradoxical that, forgetting almost all the information about the coefficients of a difference equation, we still keep the information about the (anti)periodicity of solutions.

4.3. Comparison with the classical Gale transform. Recall that the classical Gale transform of configurations of points in projective spaces is a map:

$$
\mathcal{G}_{\text {class }}: \mathcal{C}_{k+1, n} \stackrel{\simeq}{\longrightarrow} \mathcal{C}_{w+1, n},
$$

where $n=k+w+2$; see $[6,7,14,17]$.

The classical Gale transform is defined as follows. Let $A$ be a $(k+1) \times n$-matrix representing an element of $\mathcal{C}_{k+1, n}$, and let $A^{\prime}$ be a $(w+1) \times n$-matrix representing an element of $\mathcal{C}_{w+1, n}$; see (3.6) and (3.8). These elements are in Gale duality if there exists a nondegenerate diagonal $n \times n$-matrix $D$ such that

$$
A D A^{\prime T}=0 \text {, }
$$

where $A^{\prime T}$ is the transposed matrix. This is precisely the duality of the corresponding Grassmannians combined with the quotient (3.8).

To understand the difference between the combinatorial Gale transform and the classical Gale transform, recall that the space $\mathcal{E}_{k+1, n} \simeq \mathcal{F}_{k+1, n}$ is a subvariety of the Grassmannian $\mathrm{Gr}_{k+1, n}$. Given an $\mathrm{SL}_{k+1}$-frieze pattern $F$, the $(k+1) \times n$-matrix $M_{F}^{(i)}$ representing $F$, as in (2.5), satisfies the condition that every $(k+1) \times(k+1)$ minor 


$$
\Delta_{I}\left(M_{F}^{(i)}\right)=1 .
$$

This implies that the diagonal matrix $D$ also has to be of a particular form.

Proposition 4.3.1. Let $F$ be an $\mathrm{SL}_{k+1}$-frieze pattern and $\mathcal{G}(F)$ its Gale dual $\mathrm{SL}_{w+1}$-frieze pattern. Then the corresponding matrices satisfy

$$
M_{F}^{(i)} D M_{\mathcal{G}(F)}^{(j)}{ }^{T}=0
$$

where $i-j=w+1 \bmod n$, and where $D$ is the diagonal matrix

$$
D=\left(\begin{array}{cccc}
1 & 0 & \ldots & 0 \\
0 & -1 & \ldots & 0 \\
\vdots & \vdots & \ddots & \vdots \\
0 & 0 & \ldots & (-1)^{n-1}
\end{array}\right)
$$

Proof. The matrices are explicitly given by

$$
M_{F}^{(i)}=\left(\begin{array}{cccccccc}
1 & \alpha_{i-1}^{w} & & & \alpha_{i-1}^{1} & 1 & & \\
& \ddots & & & & & \ddots & \\
& & 1 & \alpha_{i+k-1}^{w} & & & \alpha_{i+k-1}^{1} & 1
\end{array}\right),
$$

of size $(k+1) \times n$, and

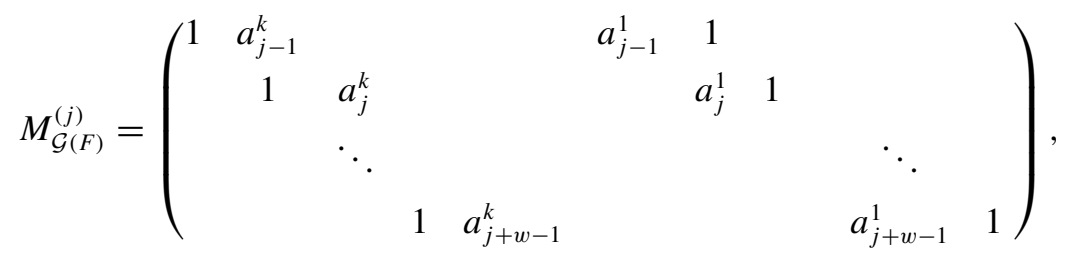

of size $(w+1) \times n$. The columns of the matrix $M_{F}^{(i)}$ correspond to the diagonals $\eta_{i-1}, \ldots, \eta_{i+k+w}$ in $F$, which are solutions of (2.1). This gives immediately the relation

$$
M_{F}^{(i)} D M_{\mathcal{G}(F)}^{(j)}{ }^{T}=0, \quad \text { where } D=\operatorname{diag}(1,-1,1,-1, \ldots) .
$$

Recall that, in Section 3.4, we defined a projection from the space of equations to the moduli space of $n$-gons. The Gale transform agrees with this projection. 

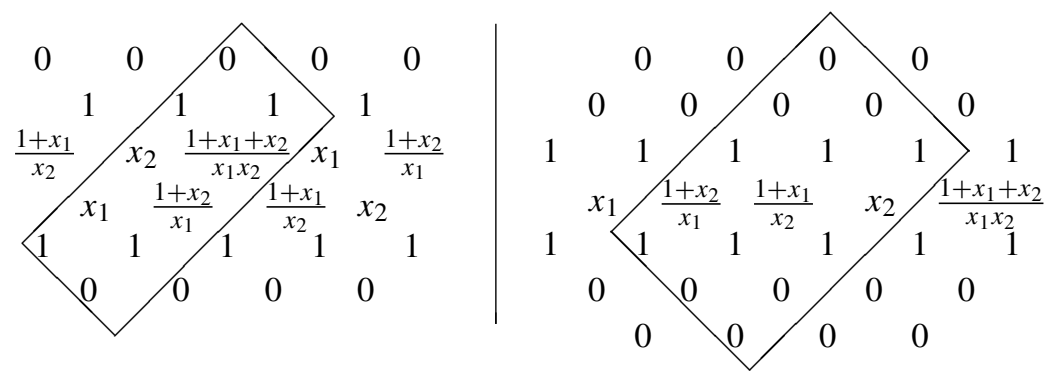

Figure 2. Gale transform between arbitrary $\mathrm{SL}_{2}$-friezes of width 2 and $\mathrm{SL}_{3}$-friezes of width 1 .

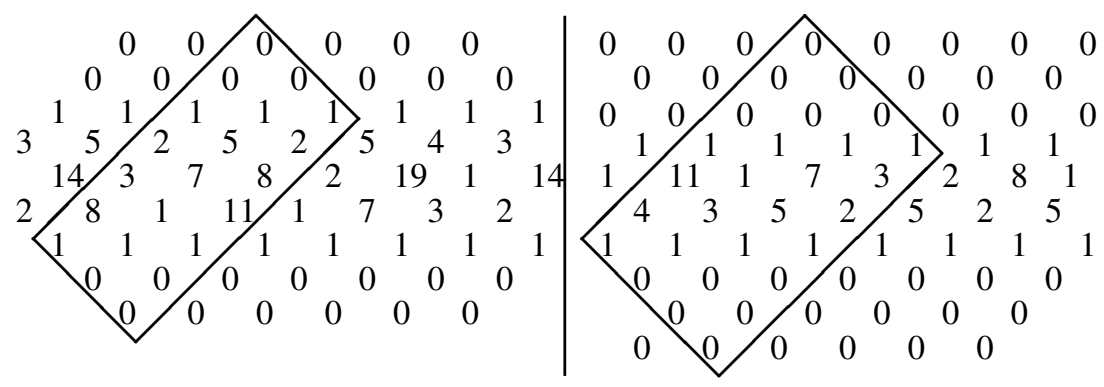

Figure 3. An $\mathrm{SL}_{3}$-frieze of width 3 and its Gale dual $\mathrm{SL}_{4}$-frieze of width 2.

COROLLARY 4.3.2. The following diagram commutes:

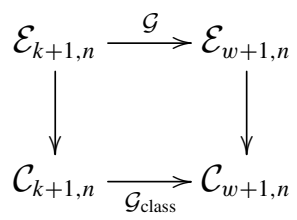

Proof. The projection $\mathcal{E}_{k+1, n} \rightarrow \mathcal{C}_{k+1, n}$, written in the matrix form, associates to a matrix representing an element of $\mathcal{E}_{k+1, n}$ a coset in the quotient $\mathrm{Gr}_{k+1, n} / \mathbb{T}^{n-1}$ defined by left multiplication by diagonal $n \times n$-matrices. If two representatives of the coset satisfy (4.17), then any two other representatives also do.

EXAMPLE 4.3.3. One immediately checks in these examples in Figures 2 and 3 that the corresponding matrices (matrix $A$ on the left and matrix $A^{\prime}$ on the right) 
satisfy

$$
A D A^{\prime T}=0, \quad \text { for } D=\operatorname{diag}(1,-1,1,-1,1,-1,1) .
$$

The Gale dual of a frieze can be obtained by computing minors in the initial frieze. For instance, in Figure 3, the first nontrivial row of the frieze on the right coincides with the last row of the frieze on the left (that is, $1 \times 1$ minors), and the second row of the frieze on the right consists of $2 \times 2$-minors formed by the last and the one before the last nontrivial rows:

$$
\left|\begin{array}{ll}
8 & 3 \\
1 & 1
\end{array}\right|, \quad\left|\begin{array}{ll}
1 & 7 \\
1 & 11
\end{array}\right|, \quad\left|\begin{array}{rr}
11 & 8 \\
1 & 1
\end{array}\right|, \quad\left|\begin{array}{ll}
1 & 2 \\
1 & 7
\end{array}\right|, \quad\left|\begin{array}{ll}
7 & 19 \\
1 & 3
\end{array}\right|, \ldots
$$

The same rule applies for going from right to left.

4.4. The projective duality. Recall that the dual projective space $\left(\mathbb{R P}^{k}\right)^{*}$ (which is of course itself isomorphic to $\mathbb{R} \mathbb{P}^{k}$ ) is the space of hyperplanes in $\mathbb{R} \mathbb{P}^{k}$. The notion of projective duality is central in projective geometry.

Projective duality is usually defined for generic $n$-gons as follows. Given an $n$-gon $\left(v_{i}\right)$ in $\mathbb{R P}^{k}$, the projectively dual $n$-gon $\left(v_{i}^{*}\right)$ in $\left(\mathbb{R} \mathbb{P}^{k}\right)^{*}$ is the $n$-gon such that each vertex $v_{i}^{*}$ is the hyperplane $\left(v_{i}, v_{i+1}, \ldots, v_{i+k-1}\right) \subset \mathbb{R P}^{k}$. This procedure obviously commutes with the action of $\mathrm{SL}_{k+1}$, so that one obtains a map

$$
\text { * : } \mathcal{C}_{k+1, n} \rightarrow \mathcal{C}_{k+1, n},
$$

which squares to a shift: $* \circ *:\left(v_{i}\right) \mapsto\left(v_{i+k-1}\right)$.

In this section, we introduce an analog of the projective duality on the space of difference equations and that of frieze patterns:

$$
*: \mathcal{E}_{k+1, n} \rightarrow \mathcal{E}_{k+1, n}, \quad *: \mathcal{F}_{k+1, n} \rightarrow \mathcal{F}_{k+1, n} .
$$

The square of $*$ also shifts the indices, but this shift is 'invisible' on equations and friezes so that it is an involution: $* \circ *=\mathrm{id}$.

DEFINITION 4.4.1. The difference equation

$$
V_{i}^{*}=a_{i+k-1}^{k} V_{i-1}^{*}-a_{i+k-2}^{k-1} V_{i-2}^{*}+\cdots+(-1)^{k-1} a_{i}^{1} V_{i-k}^{*}+(-1)^{k} V_{i-k-1}^{*}
$$

is called the projective dual of (2.1).

Here, $\left(V_{i}^{*}\right)$ is just a notation for the unknown.

EXAMPLE 4.4.2. The projective dual of the equation $V_{i}=a_{i} V_{i-1}-b_{i} V_{i-2}+V_{i-3}$ is

$$
V_{i}^{*}=b_{i+1} V_{i-1}^{*}-a_{i} V_{i-2}^{*}+V_{i-3}^{*} .
$$


The above definition is justified by the following statement.

Proposition 4.4.3. The map $\mathcal{E}_{k+1, n} \rightarrow \mathcal{C}_{k+1, n}$ from Section 3.4 commutes with projective duality.

Proof. Recall that an $n$-gon $\left(v_{i}\right)$ is in the image of the map $\mathcal{E}_{k+1, n} \rightarrow \mathcal{C}_{k+1, n}$ if and only if it is a projection of an $n$-gon $\left(V_{i}\right)$ in $\mathbb{R}^{k+1}$ satisfying the determinant condition (3.10).

Let us first show that the dual $n$-gon $\left(v_{i}^{*}\right)$ is also in the image of the map $\mathcal{E}_{k+1, n} \rightarrow \mathcal{C}_{k+1, n}$. Indeed, by the definition of projective duality, the affine coordinates of a vertex $v_{i}^{*} \in\left(\mathbb{R P}^{k}\right)^{*}$ of the dual $n$-gon can be calculated as the $k \times k$-minors of the $k \times(k+1)$-matrix

$$
\left(V_{i} V_{i+1} \ldots V_{i+k-1}\right),
$$

where $V_{j}$ are understood as $(k+1)$-vectors (that is, the columns of the matrix). Denote by $V_{i}^{*}$ the vector in $\left(\mathbb{R}^{k+1}\right)^{*}$ with coordinates given by the $k \times k$-minors. In other words, the vector $V_{i}^{*}$ is defined by the equation

$$
\left|V_{i}, V_{i+1}, \ldots, V_{i+k-1}, V_{i}^{*}\right|=1 \text {. }
$$

A direct verification then shows that the sequence $\left(V_{i}^{*}\right)$ satisfy Equation (4.19).

The isomorphism $\mathcal{E}_{k+1, n} \simeq \mathcal{F}_{k+1, n}$ allows us to define the notion of projective duality on $\mathrm{SL}_{k+1}$-frieze patterns.

Proposition 4.4.4. The projective duality of $\mathrm{SL}_{k+1}$-frieze patterns is just the symmetry with respect to the median horizontal axis.

We will prove this statement in Section 5.4. The proof uses explicit computations. See Proposition 5.4.1.

COROLLARY 4.4.5. The projective duality commutes with the Gale transform:

$$
* \circ \mathcal{G}=\mathcal{G} \circ *
$$

4.5. The self-dual case. An interesting class of difference equations and, equivalently, of $\mathrm{SL}_{k+1}$-frieze patterns, is the class of self-dual equations. In the case of frieze patterns, self-duality means invariance with respect to the horizontal axis of symmetry. 
EXAMPLE 4.5.1. (a) Every $\mathrm{SL}_{2}$-frieze pattern is self-dual.

(b) Consider the following $\mathrm{SL}_{3}$-frieze patterns of width 2:

$\begin{array}{lllllllllllllllll}1 & & 1 & & 1 & & 1 & & & 1 & & 1 & & 1 & & 1 & \\ & 2 & & 2 & & 2 & & 2 & & & 2 & & 3 & & 2 & & 3 \\ 2 & & 2 & & 2 & & 2 & & & 1 & & 5 & & 1 & & 5 & \\ & 1 & & 1 & & 1 & & 1 & & & 1 & & 1 & & 1 & & 1\end{array}$

The first one is self-dual but the second one is not.

An $n$-gon is called projectively self-dual if, for some fixed $0 \leqslant \ell \leqslant n-1$, the $n$-gon $\left(v_{i+\ell}^{*}\right)$ is projectively equivalent to $\left(v_{i}\right)$. Note that $\ell$ is a parameter in the definition (so, more accurately, one should say ' $\ell$-self-dual'); see [15].

\section{The determinantal formulas}

In this section, we give explicit formulas for the Gale transform. It turns out that one can solve Equation (2.1) and obtain explicit formulas for the coefficients of the $\mathrm{SL}_{k+1}$-frieze pattern. Let us mention that the determinant formulas presented here already appeared in the classical literature on difference equations in the context of the 'André method of solving difference equations'; see [1, 22].

5.1. Calculating the entries of the frieze patterns. Recall that we constructed an isomorphism between the spaces of difference equations $\mathcal{E}_{k+1, n}$ and frieze patterns $\mathcal{F}_{k+1, n}$. We associated an $\mathrm{SL}_{k+1}$-frieze pattern of width $w$ to every difference equation (2.1). The entries $d_{i, i+j}$ of the $\mathrm{SL}_{k+1}$-frieze pattern (also denoted by $\alpha_{i-1}^{w-j}$; see (3.12), (3.13)) were defined nonexplicitly by (3.9).

Proposition 5.1.1. The entries of the $\mathrm{SL}_{k+1}$-frieze pattern associated to a difference equation (2.1) are expressed in terms of the coefficients $a_{i}^{j}$ by the following determinants.

(i) If $0 \leqslant j \leqslant k-1$ and $j<w$, then

$$
d_{i, i+j}=\alpha_{i-1}^{w-j}=\left|\begin{array}{llll}
a_{i}^{1} & 1 & & \\
a_{i+1}^{2} & a_{i+1}^{1} & 1 & \\
\vdots & \ddots & \ddots & 1 \\
a_{i+j}^{j+1} & \cdots & a_{i+j}^{2} & a_{i+j}^{1}
\end{array}\right| .
$$


(ii) If $k-1<j<w$, then

$$
d_{i, i+j}=\alpha_{i-1}^{w-j}=\left|\begin{array}{cccccc}
a_{i}^{1} & 1 & & & & \\
\vdots & a_{i+1}^{1} & 1 & & & \\
a_{i+k-1}^{k} & & \ddots & \ddots & & \\
1 & & & \ddots & \ddots & \\
& \ddots & & & a_{i+j-1}^{1} & 1 \\
& & 1 & a_{i+j}^{k} & \ldots & a_{i+j}^{1}
\end{array}\right| .
$$

Proof. We use the Gale transform $\mathcal{G}(F)$ of the frieze $F$ associated to (2.1). In $\mathcal{G}(F)$, the following diagonals of length $w+1$

$$
\begin{aligned}
& \begin{array}{lllll}
\eta_{i-w-2} & \eta_{i-w-1} & \eta_{i-w+j} & \eta_{i-2} & \eta_{i-1}
\end{array} \\
& \left(\begin{array}{ccccccc}
a_{i}^{1} & 1 & & & & & \\
a_{i+1}^{2} & a_{i+1}^{1} & \ddots & & & & \\
\vdots & \vdots & & 1 & & & \\
a_{i+j}^{j+1} & a_{i+j}^{j} & & a_{i+j}^{1} & \ddots & & \\
\vdots & \vdots & & \vdots & & 1 & \\
\vdots & \vdots & & \vdots & & a_{i+w}^{1} & 1
\end{array}\right),
\end{aligned}
$$

satisfy the recurrence relation

$$
\begin{aligned}
\eta_{i-1}= & \alpha_{i-1}^{1} \eta_{i-2}-\cdots+(-1)^{w-j-1} \alpha_{i-1}^{w-j} \eta_{i-w+j-1} \\
& +\cdots+(-1)^{w-1} \alpha_{i-1}^{w} \eta_{i-w-1}+(-1)^{w} \eta_{i-w-2},
\end{aligned}
$$

which can be written in terms of vectors and matrices as

$$
\eta_{i-1}=\left(\begin{array}{lll}
\eta_{i-w-2}, & \ldots, & \eta_{i-2}
\end{array}\right)\left(\begin{array}{l}
(-1)^{w} \\
(-1)^{w-1} \alpha_{i-1}^{w} \\
\vdots \\
(-1)^{0} \alpha_{i-1}^{1}
\end{array}\right) .
$$


The coefficients $\alpha_{i-1}^{w-j}$ can be computed using the Cramer rule,

$$
\alpha_{i-1}^{w-j}=\frac{(-1)^{w-j-1}\left|\eta_{i-w-2}, \ldots, \eta_{i-w+j}, \eta_{i-1}, \eta_{i-w+j+2}, \ldots, \eta_{i-2}\right|}{\left|\eta_{i-w-2}, \ldots, \eta_{i-2}\right|}
$$

The denominator is 1 , since $\mathcal{G}(F)$ is an $\mathrm{SL}_{w+1}$-frieze, and the numerator simplifies to (5.20) or to (5.21) accordingly after decomposing by the $\eta_{i-1}$ th column. The coefficient $\alpha_{i-1}^{w-j}$ is in position $d_{i, i+j}$ in the frieze $F$.

5.2. Equivalent formulas. There is another, alternative, way to calculate the entries of the $\mathrm{SL}_{k+1}$-frieze pattern.

Proposition 5.2.1. (i) If $j+k \geqslant w$, then

$$
d_{i, i+j}=\left|\begin{array}{ccccc}
a_{i-w+j-1}^{k} & a_{i-w+j-1}^{k-1} & \ldots & \ldots & a_{i-w+j-1}^{k-w+j+1} \\
1 & a_{i-w+j}^{k} & \ldots & \ldots & a_{i-w+j}^{k-w+j} \\
& 1 & & & \vdots \\
& & \ddots & \ddots & \vdots \\
& & & & \\
& & & & a_{i-2}^{k}
\end{array}\right| .
$$

(ii) If $j+k<w$, then

$$
d_{i, i+j}=\left|\begin{array}{cccccc}
a_{i-w+j-1}^{k} & \ldots & a_{i-w+j-1}^{1} & 1 & & \\
1 & a_{i-w+j}^{k} & \ldots & a_{i-w+j}^{1} & 1 & \\
1 & \ldots & \ldots & & 1 \\
& \ddots & & \ddots & \vdots \\
& & 1 & a_{i-3}^{k} & a_{i-3}^{k-1} \\
& & & 1 & a_{i-2}^{k}
\end{array}\right|
$$

Proof. These formulas are obtained in the same way as (5.20) and (5.21). 
EXAMPLE 5.2.2. (a) Hill's equations $V_{i}=a_{i} V_{i-1}-V_{i-2}$ with antiperiodic solutions correspond to Coxeter's frieze patterns with the entries

$$
d_{i, i+j}=\left|\begin{array}{ccccc}
a_{i} & 1 & & & \\
1 & a_{i+1} & 1 & & \\
& \ddots & \ddots & \ddots & \\
& & 1 & a_{i+j-2} & 1 \\
& & & 1 & a_{i+j-1}
\end{array}\right| .
$$

The corresponding geometric space is the moduli space (see Theorem 3.4.1) of $n$ gons in the projective line known (in the complex case) under the name of moduli space $\mathcal{M}_{0, n}$.

(b) In the case of third-order difference equations, $V_{i}=a_{i} V_{i-1}-b_{i} V_{i-2}+V_{i-3}$, we have

$$
d_{i, i+j}=\left|\begin{array}{cccccc}
a_{i} & b_{i+1} & 1 & & & \\
1 & a_{i+1} & b_{i+2} & 1 & & \\
& \ddots & \ddots & \ddots & \ddots & \\
& & 1 & a_{i+j-3} & b_{i+j-3} & 1 \\
& & & 1 & a_{i+j-2} & b_{i+j-2} \\
& & & & 1 & a_{i+j-1}
\end{array}\right| .
$$

This case is related to the moduli space $\mathcal{C}_{3, n}$ of $n$-gons in the projective plane studied in [32].

5.3. Determinantal formulas for the Gale transform. Formulas from Propositions 5.1.1 and 5.2.1 express the entries of the $\mathrm{SL}_{k+1}$-frieze pattern (3.12) as minors of the Gale dual $\mathrm{SL}_{w+1}$-frieze pattern (4.16). One can reverse the formulas and express the entries of (4.16), that is, the coefficients of Equation (2.1) as minors of the $\mathrm{SL}_{k+1}$-frieze pattern (3.12).

For instance, the Gale dual of (5.22) is

$$
a_{i-1}^{k-j}=\frac{(-1)^{k-j-1}\left|\eta_{i-k-2}, \ldots, \eta_{i-k+j}, \eta_{i-1}, \eta_{i-k+j+2}, \ldots, \eta_{i-2}\right|}{\left|\eta_{i-k-2}, \ldots, \eta_{i-2}\right|},
$$

where the $\eta$ are the South-East diagonals of the $\mathrm{SL}_{k+1}$-frieze pattern (3.12). Note that the denominator is equal to 1 .

For $j<k$, we obtain an analog of formula (5.20): 


$$
a_{i-1}^{k-j}=\left|\begin{array}{ccc}
d_{i+1, i+w} & 1 & \\
\vdots & \ddots & 1 \\
d_{i+j+1, i+w} & \cdots & d_{i+j+1, i+j+w}
\end{array}\right|,
$$

and similarly for (5.21).

\subsection{Frieze patterns and the dual equations.}

PROPOSITION 5.4.1. The North-East diagonals $\left(\mu_{i}\right)$ of the $\mathrm{SL}_{k+1}$-frieze pattern corresponding to the difference equation (2.1) satisfy the projectively dual difference equation (4.19).

Proof. This is a consequence of the tameness of the $\mathrm{SL}_{k+1}$-frieze pattern corresponding to (2.1) and can be easily shown using the explicit determinantal formulas (5.23) that will be established in the next section. We first consider the case $j=i+w-k-2$. We know that the $(k+2) \times(k+2)$-determinant vanishes; thus

$$
\left|\begin{array}{cccc}
d_{j-w+1, j} & 1 & & \\
d_{j-w+2, j} & d_{j-w+2, j+1} & 1 & \\
\vdots & & \ddots & 1 \\
d_{i, j} & \ldots & & d_{i, i+w-1}
\end{array}\right|=0 .
$$

Decomposing the determinant by the first column gives the recurrence relation

$$
d_{i, j}=a_{i-2}^{k} d_{i-1, j}-a_{i-3}^{k-1} d_{i-1, j}+\cdots+(-1)^{k-1} a_{i-k-1}^{1} d_{i-k, j}+(-1)^{k} d_{i-k-1, j},
$$

where the coefficients are computed using (5.23). The recurrence relation then propagates inside the frieze due to the tameness property, and hence will hold for all $j$. This proves that the North-East diagonals satisfy (4.19) after renumbering $\mu_{i}^{\prime}:=\mu_{i+k+1}$.

\section{The Gale transform and representation theory}

In this section, we give another description of the combinatorial Gale transform $\mathcal{G}$ in terms of representation theory of the Lie group $\mathrm{SL}_{n}$. Let $N \subset \mathrm{SL}_{n}$ be the subgroup of upper unitriangular matrices. 


$$
A=\left(\begin{array}{cccc}
1 & * & \ldots & * \\
& \ddots & \ddots & \vdots \\
& & \ddots & * \\
& & & 1
\end{array}\right) .
$$

We will associate a unitriangular $n \times n$-matrix to every $\mathrm{SL}_{k+1}$-frieze pattern. This idea allows us to apply in our situation many tools of the theory of matrices, as well as more sophisticated tools of representation theory. We make just one small step in this direction: we show that the combinatorial Gale transform $\mathcal{G}$ coincides with the restriction of the antiinvolution on $N$ introduced in [3].

6.1. From frieze patterns to unitriangular matrices. Given an $\mathrm{SL}_{k+1}$-frieze pattern $F$ of width $w$, as in formula (2.3), cutting out a piece of the frieze, see Figure 4 , we associate to $F$ a unitriangular $n \times n$-matrix

$$
A_{F}=\left(\begin{array}{ccccccc}
1 & d_{1,1} & \cdots & d_{1, w} & 1 & & \\
& \ddots & \ddots & & \ddots & \ddots & \\
& & \ddots & \ddots & \ddots & 1 \\
& & \ddots & \ddots & & d_{n-w, n-1} \\
& & & \ddots & \ddots & \vdots \\
& & & & & \ddots & d_{n-1, n-1} \\
& & & & & & 1
\end{array}\right)
$$

with $w+2$ nonzero diagonals. As before, $n=k+w+2$.

REMARK 6.1.1. Note that the matrix $A_{F}$ contains all the information about the $\mathrm{SL}_{k+1}$-frieze pattern. Indeed, the frieze pattern can even be reconstructed by a much smaller $(k+1) \times n$-matrix (2.5). However, $A_{F}$ is not defined uniquely.Indeed, it depends on the choice of the first element $d_{1,1}$ in the first line of the frieze. A different cutting gives a different matrix.

6.2. The combinatorial Gale transform as an antiinvolution on $N$. Denote by ' the antiinvolution of $N$ defined for $x \in N$ by

$$
x^{\iota}=D x^{-1} D,
$$

where $D$ is the diagonal matrix (4.18). Recall that the term 'antiinvolution' means an involution that is an antihomomorphism; that is, $(x y)^{\iota}=y^{\iota} x^{\iota}$. 


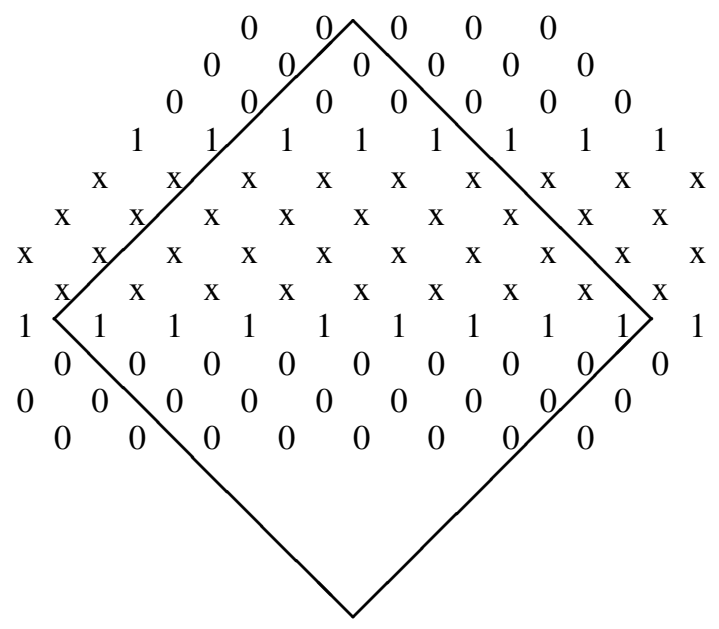

Figure 4. Cutting a unitriangular matrix out of a frieze.

This antiinvolution was introduced in [3] in order to study the canonical parameterizations of $N$. We will explain the relation of ${ }^{\prime}$ to the classical representation theory in Section 6.4.

THEOREM 6.2.1. (i) The operation $A_{F} \mapsto\left(A_{F}\right)^{\iota}$ associates to a matrix (6.25) of a tame $\mathrm{SL}_{k+1}$-frieze pattern a matrix of a tame $\mathrm{SL}_{w+1}$-frieze pattern.

(ii) The corresponding map

$$
{ }^{\imath}: \mathcal{E}_{k+1, n} \rightarrow \mathcal{E}_{w+1, n}
$$

coincides with the composition of the Gale transform and the projective duality:

$$
{ }^{\imath}=\mathcal{G} \circ * .
$$

Proof. Let us denote by $S=\{1,2, \ldots, n\}$ the index set of the rows, respectively columns, of a matrix $x \in N$. For two subsets $I, J \subset S$ of the same cardinality, we denote by $\Delta_{I, J}(x)$ the minor of the matrix $x$ taken over the rows of indices in $I$ and the columns of indices in $J$. We have the following well-known relation:

$$
\Delta_{i, j}\left(x^{\iota}\right)=\Delta_{S-\{j\}, S-\{i\}}(x),
$$

where $\Delta_{i, j}\left(x^{\iota}\right)$ is simply the entry in position $(i, j)$ in $x^{\iota}$. Taking into account that the matrix $x$ belongs to $N$, whenever $j>i$, the $(n-1) \times(n-1)$-minor in the above right-hand side simplifies to a $(j-i) \times(j-i)$-minor 


$$
\Delta_{i, j}\left(x^{\iota}\right)=\Delta_{[i, j-1],[i+1, j]}(x),
$$

where $[a, b]$ denotes the interval $\{a, a+1, \ldots, b\}$. Let us use this relation to compute the entry in position $(i, j)$ in $\left(A_{F}\right)^{\iota}$. One has

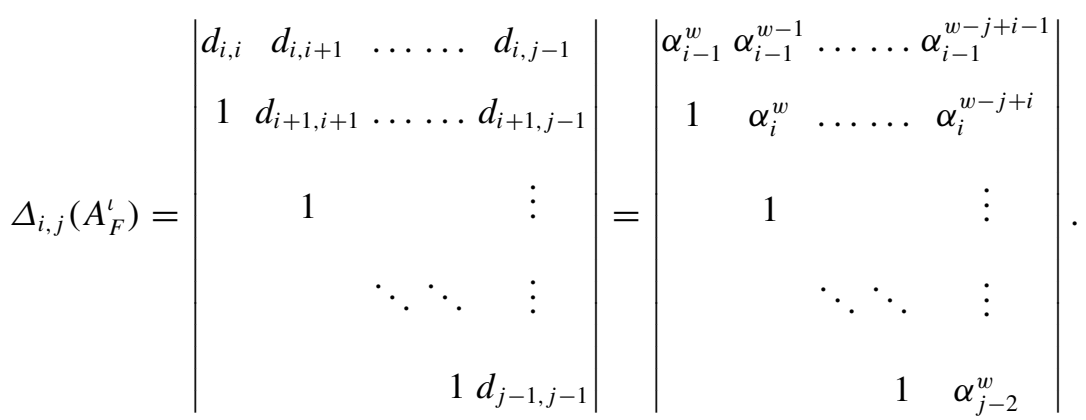

According to the determinantal formulas of Section 5, this is precisely the entry $d_{j, i+k}$ of the frieze $\mathcal{G}(F)$, and the result follows.

6.3. Elements of the representation theory. Our next goal is to explain the relation of the involution ' with Schubert cells in the Grassmannians. We have to recall some basic notions of representation theory.

The Weyl group of $\mathrm{SL}_{n}$ is the group $\mathcal{S}_{n}$ of permutations over $n$ letters that we think of as the set of integers $\{1,2, \ldots, n\}$. The group $\mathcal{S}_{n}$ is generated by $(n-1)$ elements denoted by $s_{i}, 1 \leqslant i \leqslant n-1$, representing the elementary transposition $i \leftrightarrow i+1$. The relations between the generators are as follows:

$$
\begin{aligned}
s_{i} s_{i+1} s_{i} & =s_{i+1} s_{i} s_{i+1}, \\
s_{i} s_{j} & =s_{j} s_{i}, \quad|i-j|>1 .
\end{aligned}
$$

A decomposition of $\sigma \in \mathcal{S}_{n}$ as

$$
\sigma=s_{i_{1}} s_{i_{2}} \cdots s_{i_{p}}
$$

is called reduced if it involves the least possible number of generators. Equivalently, we call the sequence $\mathbf{i}=\left(i_{1}, \ldots, i_{p}\right)$ a reduced word for $\sigma$.

The group $\mathcal{S}_{n}$ can be viewed as a subgroup of $\mathrm{SL}_{n}$ using the following lift of the generators:

$$
s_{i}=\left(\begin{array}{cccc}
\ddots & & & \\
& 0 & 1 & \\
& -1 & 0 & \\
& & & \ddots
\end{array}\right) .
$$


Let us now describe the standard parameterization of the unipotent subgroup $N$. Consider the following one-parameter subgroups of $N$ :

$$
x_{i}(t)=\left(\begin{array}{llll}
\ddots & & & \\
& 1 & t & \\
& & 1 & \\
& & & \ddots
\end{array}\right), \quad 1 \leqslant i \leqslant n-1, t \in \mathbb{R},
$$

where $t$ is in position $(i, i+1)$. The matrices $x_{i}(t)$ are called the elementary Jacobi matrices; these are generators of $N$.

The next notion we need is that of the Schubert cells. Denote by $B^{-}$the Borel subgroup of lower triangular matrices of $\mathrm{SL}_{n}$. Fix an arbitrary element $\sigma \in \mathcal{S}_{n}$, and consider the set

$$
N^{\sigma}:=N \cap B^{-} \sigma B^{-},
$$

which is known as an open dense subset of a Schubert cell. It is well known that generically elements of $N^{\sigma}$ can be represented as

$$
x_{\mathbf{i}}(\mathbf{t})=x_{i_{1}}\left(t_{1}\right) x_{i_{2}}\left(t_{2}\right) \cdots x_{i_{p}}\left(t_{p}\right),
$$

where $\mathbf{t}=\left(t_{1}, \ldots, t_{p}\right) \in \mathbb{R}^{p}$ and $\mathbf{i}=\left(i_{1}, \ldots, i_{p}\right)$ is an arbitrary reduced word for $\sigma$.

For the choice

$$
\sigma=s_{k+1} \cdots s_{n-1} s_{k} \cdots s_{n-2} \cdots s_{1} \cdots s_{n-k-1},
$$

the set $N^{\sigma}$ is identified with an open subset of the Grassmannian $\mathrm{Gr}_{k+1, n}$. See, for example, [44, Ch. 8], for more details.

6.4. The antiinvolution ' and the Grassmannians. It was proved in [3] that the antiinvolution ' on $N$ can be written in terms of the generators as follows. Set $x_{i}(t)^{\iota}=x_{i}(t)$; then, for $x$ as in (6.27), one has

$$
x^{\iota}=x_{i_{p}}\left(t_{p}\right) x_{i_{p-1}}\left(t_{p-1}\right) \cdots x_{i_{1}}\left(t_{1}\right) .
$$

This map is well defined; that is, it is independent of the choice of the decomposition of $x$ into a product of generators, since it coincides with (6.26).

Restricted to $N^{\sigma}$, where $\sigma$ is given by (6.28), the antiinvolution reads ${ }^{\imath}: N^{\sigma} \rightarrow$ $N^{\sigma^{-1}}$. In particular, it sends an open subset of the Grassmannian $\mathrm{Gr}_{k+1, n}$ to an open subset of $\mathrm{Gr}_{w+1, n}$. 
We have already defined the embedding (2.5) of the space of $\mathrm{SL}_{k+1}$-frieze patterns into the Grassmannian. Quite obviously, one also has an embedding into $N^{\sigma}$, so that

$$
\mathcal{F}_{k+1, n} \subset N^{\sigma} \subset \mathrm{Gr}_{k+1, n} .
$$

It can be shown that the image of the involution ' restricted to $\mathcal{F}_{k+1, n}$ belongs to $\mathcal{F}_{w+1, n}$. However, the proof is technically involved, and we do not dwell on the details here. The following example illustrates the situation quite well.

EXAMPLE 6.4.1. The case of $\mathrm{Gr}_{2,5}$. Fix $\sigma=s_{2} s_{3} s_{4} s_{1} s_{2} s_{3}$, and consider the following element of $N^{\sigma}$ :

$$
x=x_{2}\left(t_{1}\right) x_{3}\left(t_{2}\right) x_{4}\left(t_{3}\right) x_{1}\left(t_{4}\right) x_{2}\left(t_{5}\right) x_{3}\left(t_{6}\right)=\left(\begin{array}{cccc}
1 t_{4} & t_{4} t_{5} & t_{4} t_{5} t_{6} & 0 \\
1 & t_{5}+t_{1} & t_{1} t_{2}+t_{1} t_{6}+t_{5} t_{6} & t_{1} t_{2} t_{3} \\
& 1 & t_{6}+t_{2} & t_{2} t_{3} \\
& 1 & t_{3} \\
& & 1
\end{array}\right) .
$$

One then has

$$
x^{\iota}=x_{3}\left(t_{6}\right) x_{2}\left(t_{5}\right) x_{1}\left(t_{4}\right) x_{4}\left(t_{3}\right) x_{3}\left(t_{2}\right) x_{2}\left(t_{1}\right)=\left(\begin{array}{ccccc}
1 & t_{4} & t_{4} t_{1} & 0 & 0 \\
& 1 & t_{5}+t_{1} & t_{2} t_{5} & 0 \\
& & 1 & t_{6}+t_{2} & t_{6} t_{3} \\
& & & 1 & t_{3} \\
& & & & 1
\end{array}\right) .
$$

If now $x \in \mathcal{F}_{2,5}$, so that every $2 \times 2$-minor equals 1 , then one has, after an easy computation,

$$
t_{1} t_{4}=1, \quad t_{1} t_{2} t_{4} t_{5}=1, \quad t_{1} t_{2} t_{3} t_{4} t_{5} t_{6}=1, \quad t_{1} t_{2} t_{3}=1,
$$

and this implies that $x^{\iota} \in \mathcal{F}_{3,5}$.

\section{Periodic rational maps from frieze patterns}

The periodic rational maps described in this section are a simple consequence of the isomorphism $\mathcal{E}_{k+1, n} \simeq \mathcal{F}_{k+1, n}$ and of the periodicity condition. However, the maps are of interest. The simplest example is known as the Gauss map; see [18]. This map is given explicitly by 


$$
\left(c_{1}, c_{2}\right) \mapsto\left(c_{2}, \frac{1+c_{1}}{c_{1} c_{2}-1}\right),
$$

where $c_{1}, c_{2}$ are variables. Gauss proved that this map is 5-periodic. In our terminology, the Gauss map consists in the index shift $\left(c_{1}, c_{2}\right) \mapsto\left(c_{2}, c_{3}\right)$ in a Hill equation $V_{i}=c_{i} V_{i-1}-V_{i-2}$ with 5-antiperiodic solutions.

The maps that we calculate in Section 7.1 are related to the so-called Zamolodchikov periodicity conjecture. They can be deduced from the simplest $A_{k} \times A_{w}$-case; the periodicity was proved in this case in [45] by a different method. The general case of the conjecture was recently proved in [24]. It would be interesting to investigate an approach based on linear difference equations in this case.

Finally, the maps that we calculate in Section 7.2 correspond to self-dual difference equations. They do not enter into the framework of the Zamolodchikov periodicity conjecture, and seem to be new.

7.1. Periodicity of $\mathrm{SL}_{k+1}$-frieze patterns and generalized Gauss maps. An important property of $\mathrm{SL}_{k+1}$-frieze patterns is their periodicity.

COROLlaRY 7.1.1. Tame $\mathrm{SL}_{k+1}$-frieze patterns of width $w$ are n-periodic in the horizontal direction: $d_{i, j}=d_{i+n, j+n}$ for all $i, j$, where $n=k+w+2$.

This statement is a simple corollary of Theorem 3.4.1, Part (i). Note that, in the simplest case $k=1$, the above statement was proved by Coxeter [9].

Let us introduce the following notation:

$$
U\left(a_{1}, a_{2}, \ldots, a_{k}\right):=\left|\begin{array}{ccccc}
a_{1} & 1 & & & \\
1 & a_{2} & 1 & & \\
& \ddots & \ddots & \ddots & \\
& & 1 & a_{k-1} & 1 \\
& & & 1 & a_{k}
\end{array}\right|
$$

for the simplest determinants from Section 5; see Example 5.2.2, part (a). We obtain the following family of rational periodic maps.

COROLlaRY 7.1.2. Let the rational map $F: \mathbb{R}^{n-3} \rightarrow \mathbb{R}^{n-3}$ be given by the formula

$$
F:\left(a_{1}, a_{2}, \ldots, a_{n-3}\right) \mapsto\left(a_{2}, a_{3}, \ldots, a_{n-3}, P\left(a_{1}, a_{2}, \ldots, a_{n-3}\right)\right),
$$


where

$$
P\left(a_{1}, a_{2}, \ldots, a_{n-3}\right)=\frac{1+U\left(a_{1}, \ldots, a_{n-4}\right)}{U\left(a_{1}, \ldots, a_{n-3}\right)}
$$

Then $F^{n}=\mathrm{id}$.

Proof. Consider the periodic Hill equation $V_{i}=a_{i} V_{i-1}-V_{i-2}$, or equivalently the $\mathrm{SL}_{2}$-frieze pattern whose first row consists of $1 \mathrm{~s}$, and whose second row is the biinfinite sequence $\left(a_{i}\right)$. The entries of the $k$ th row of this frieze pattern are

$$
U\left(a_{i}, \ldots, a_{i+k-2}\right), \quad i \in \mathbb{Z}
$$

see Example 5.2.2 and [9, 32].

Assume that all the solutions of the Hill equation are $n$-antiperiodic. Then the frieze pattern is closed, of width $n-3$, and its rows are $n$-periodic; see [9] and Corollary 7.1.1. Furthermore, the $(n-2)$ th row of a closed $\mathrm{SL}_{2}$-frieze pattern consists of $1 \mathrm{~s}$. Therefore

$$
U\left(a_{i}, a_{i+1}, \ldots, a_{i+n-3}\right)=1,
$$

for all $i$.

On the other hand, decomposing the determinant $U\left(a_{i}, a_{i+1}, \ldots, a_{i+n-3}\right)$ by the last row, we find

$$
a_{i+n-3}=P\left(a_{i}, \ldots, a_{i+n-4}\right) \text {. }
$$

We can choose $a_{1}, \ldots, a_{n-3}$ arbitrarily, and then consecutively define $a_{n-2}, a_{n-1}$, ... using formula (7.29) for $i=1,2, \ldots$ That is, we reconstruct the sequence $\left(a_{i}\right)$ from the 'seed' $\left\{a_{1}, \ldots, a_{n-3}\right\}$ by iterating the map $F$. By the periodicity assumption, the result is an $n$-periodic sequence.

Introduce another notation:

$$
V\left(a_{1}, b_{1}, \ldots, b_{k-1}, a_{k}\right):=\left|\begin{array}{cccccc}
a_{1} & b_{1} & 1 & & & \\
1 & a_{2} & b_{2} & 1 & & \\
& \ddots & \ddots & \ddots & \ddots & \\
& & 1 & a_{k-2} & b_{k-2} & 1 \\
& & & 1 & a_{k-1} & b_{k-1} \\
& & & & 1 & a_{k}
\end{array}\right|
$$

see Example 5.2.2, part (b). 
COROLlaRY 7.1.3. Let the rational map $\Phi: \mathbb{R}^{2 n-8} \rightarrow \mathbb{R}^{2 n-8}$ be given by the formula

$$
\begin{aligned}
\Phi & :\left(a_{1}, b_{1}, a_{2}, b_{2}, \ldots, a_{n-4}, b_{n-4}\right) \\
& \mapsto\left(b_{1}, a_{2}, b_{2}, \ldots, b_{n-4}, Q\left(a_{1}, b_{1}, \ldots, b_{n-4}, a_{n-4}\right)\right),
\end{aligned}
$$

where

$$
Q\left(a_{1}, b_{1}, \ldots, a_{n-4}, b_{n-4}\right)=\frac{1+b_{n-4} V\left(a_{1}, b_{1}, \ldots, a_{n-5}\right)-V\left(a_{1}, b_{1}, \ldots, a_{n-6}\right)}{V\left(a_{1}, b_{1}, \ldots, a_{n-4}\right)} .
$$

Then $\Phi^{2 n}=\mathrm{id}$.

Proof. The arguments are similar to those of the above proof, but we will also use the notion of a projectively dual equation.

Consider the difference equation $V_{i}=a_{i} V_{i-1}-b_{i} V_{i-2}+V_{i-3}$, and assume that all its solutions (and therefore all its coefficients) are $n$-periodic. Consider the dual equation, see Example 4.4.2, but 'read' it from right to left:

$$
V_{i-3}^{*}=a_{i} V_{i-2}^{*}-b_{i+1} V_{i-1}^{*}+V_{i}^{*} \text {. }
$$

The map $\Phi$ associates to a 'seed' $\left\{a_{1}, b_{1}, \ldots, a_{n-4}, b_{n-4}\right\}$ of the initial equation the same 'seed' of the dual equation.

Recall finally that the double iteration of the projective duality is a shift: $i \rightarrow$ $i+1$. Therefore, $\Phi^{2}:\left(a_{i}, b_{i}, \ldots\right) \mapsto\left(a_{i+1}, b_{i+1}, \ldots\right)$, which is $n$-periodic by assumption.

EXAMPLE 7.1.4. For $n=5$, the maps from Corollaries 7.1.2 and 7.1.3 are as follows:

$$
F\left(a_{1}, a_{2}\right)=\left(a_{2}, \frac{1+a_{1}}{a_{1} a_{2}-1}\right), \quad \Phi(b, a)=\left(a, \frac{a+1}{b}\right) .
$$

The first one is the classical 5-periodic Gauss map; the second, which looks even more elementary, is 10-periodic.

7.2. Periodic maps in the self-dual case. Let us now consider a version of the rational periodic maps that correspond to self-dual third-order $n$-periodic equations; see Section 4.5.

COROLlaRY 7.2.1. (i) Let $n=2 m-1$, and let the rational map $G_{o}: \mathbb{R}^{n-4} \rightarrow$ $\mathbb{R}^{n-4}$ be given by the formula

$$
\begin{aligned}
& G_{o}\left(a_{1}, b_{1}, a_{2}, b_{2}, \ldots, a_{m-2}, b_{m-2}\right) \\
& \quad=\left(b_{1}, a_{2}, b_{2}, \ldots, b_{m-2}, R_{o}\left(a_{1}, b_{1}, \ldots, a_{m-2}, b_{m-2}\right)\right),
\end{aligned}
$$


where

$$
\begin{aligned}
& R_{o}\left(a_{1}, b_{1}, \ldots, a_{m-2}, b_{m-2}\right) \\
& \quad=\frac{V\left(a_{2}, b_{2}, \ldots, a_{m-2}\right)+b_{m-2} V\left(a_{1}, b_{1}, \ldots, a_{m-3}\right)-V\left(a_{1}, b_{1}, \ldots, a_{m-4}\right)}{V\left(a_{1}, b_{1}, \ldots, a_{m-2}\right)} .
\end{aligned}
$$

Then $G_{o}^{n}=\mathrm{id}$.

(ii) Let $n=2 m$, and let the rational map $G_{e}: \mathbb{R}^{n-4} \rightarrow \mathbb{R}^{n-4}$ be given by the formula

$$
\begin{aligned}
& G_{e}\left(a_{1}, b_{1}, a_{2}, b_{2}, \ldots, a_{m-2}, b_{m-2}\right) \\
& \quad=\left(b_{1}, a_{2}, b_{2}, \ldots, b_{m-2}, R_{e}\left(a_{1}, b_{1}, \ldots, a_{m-2}, b_{m-2}\right)\right)
\end{aligned}
$$

where

$$
\begin{aligned}
& R_{e}\left(a_{1}, b_{1}, \ldots, a_{m-2}, b_{m-2}\right) \\
& \quad=\frac{V\left(b_{2}, a_{3}, \ldots, a_{m-2}\right)+b_{m-2} V\left(a_{1}, b_{1}, \ldots, a_{m-3}\right)-V\left(a_{1}, b_{1}, \ldots, a_{m-4}\right)}{V\left(a_{1}, b_{1}, \ldots, a_{m-2}\right)} .
\end{aligned}
$$

Then $G_{e}^{n}=\mathrm{id}$.

Proof. Let us consider part (i); the other case is similar.

As before, consider $n$-periodic equations $V_{i}=a_{i} V_{i-1}-b_{i} V_{i-2}+V_{i-3}$. As before, both sequences $\left(a_{i}\right)$ and $\left(b_{i}\right)$ are $n$-periodic, so that the sequence

$$
\ldots, b_{1}, a_{1}, b_{2}, a_{2}, \ldots, b_{n}, a_{n}, \ldots
$$

is $2 n$-periodic. However, if the equation is self-dual, then this sequence is, actually, $n$-periodic. More precisely,

$$
\begin{cases}b_{i+m}=a_{i}, & n=2 m-1 \\ b_{i+m}=b_{i}, a_{i+m}=a_{i}, & n=2 m .\end{cases}
$$

We are concerned with the shift

$$
\left(a_{1}, b_{1}, a_{2}, b_{2}, \ldots, a_{m-2}, b_{m-2}\right) \mapsto\left(b_{1}, a_{2}, b_{2}, \ldots, a_{m-2}, b_{m-2}, a_{m-1}\right),
$$

and we need to express $a_{m-1}$ as a function of $a_{1}, b_{1}, a_{2}, b_{2}, \ldots, a_{m-2}, b_{m-2}$.

To this end, we express, in two ways, the same entry of the $\mathrm{SL}_{3}$-frieze pattern. Consider the South-East diagonal through the entry $a_{1}$ of the top nontrivial row and the South-West diagonal through the entry $a_{m-1}$ of the same row. The entry at their intersection is $V\left(a_{1}, b_{1}, \ldots, a_{m-1}\right)$; see [32]. 
Since the difference equation is self-dual, the bottom nontrivial row of the $\mathrm{SL}_{3}$ frieze pattern is identical to the top one. The North-East diagonal through the entry $a_{2}$ of this bottom nontrivial row intersects the North-West diagonal through the entry $a_{m-2}$ of the same row at the same point as above, and the entry there is $V\left(a_{2}, b_{2}, \ldots, a_{m-2}\right)$.

Therefore

$$
V\left(a_{1}, b_{1}, \ldots, a_{m-1}\right)=V\left(a_{2}, b_{2}, \ldots, a_{m-2}\right),
$$

and it remains to solve this equation for $a_{m-1}$. This yields the formula for the rational function $R$.

EXAMPLE 7.2.2. If $n=6$, we obtain the map

$$
G_{e}(a, b)=\left(b, \frac{2 b}{a}\right)
$$

that indeed has order 6 . If $n=7$, we obtain the map

$$
G_{o}\left(a_{1}, b_{1}, a_{2}, b_{2}\right)=\left(b_{1}, a_{2}, b_{2}, \frac{a_{2}+a_{1} b_{2}-1}{a_{1} a_{2}-b_{1}}\right)
$$

that has order 7.

7.3. Another expression for $\mathbf{2 n}$-periodic maps. Let us sketch a derivation of the map $\Phi$ from a geometrical point of view. The formula is

$$
\Phi\left(x_{1}, \ldots, x_{2 n-8}\right)=\left(x_{2}, \ldots, x_{2 n-8}, R\left(x_{1}, \ldots, x_{2 n-8}\right)\right),
$$

where

$$
R\left(x_{1}, \ldots, x_{2 n-8}\right)=\frac{O_{-1}^{2 n-7}}{O_{-1}^{2 n-9}-x_{2 n-8} x_{2 n-9} O_{-1}^{2 n-11}},
$$

and where $O_{a}^{b}$ is defined by recurrence relation

$$
O_{a}^{b}=O_{a}^{b-2}-x_{b-2} O_{a}^{b-4}+x_{b-2} x_{b-3} x_{b-4} O_{a}^{b-6}, \quad a=b-4, b-6, \ldots
$$

with the initial conditions $O_{b}^{b}=O_{b-2}^{b}=1$. We will see below why $\Phi^{2 n}$ is the identity map.

EXAMPLE 7.3.1. The first nontrivial example is

$$
\Phi\left(x_{1}, x_{2}\right)=\left(x_{2}, \frac{1-x_{1}}{1-x_{1} x_{2}}\right),
$$


which is the Gauss map. The next example is

$$
\Phi\left(x_{1}, x_{2}, x_{3}, x_{4}\right)=\left(x_{2}, x_{3}, x_{4}, \frac{1-x_{1}-x_{3}+x_{1} x_{2} x_{3}}{1-x_{1}-x_{3} x_{4}}\right)
$$

which is 12-periodic.

These formulas give the expression of the map $\Phi$ in so-called corner coordinates on the moduli space of $n$-gons in $\mathbb{P}^{2}$. Let $L L^{\prime}$ denote the intersection of lines $L$ and $L^{\prime}$, and let $P P^{\prime}$ denote the line containing $P$ and $P^{\prime}$. We have the inverse cross-ratio

$$
[a, b, c, d]=\frac{(a-b)(c-d)}{(a-c)(b-d)} .
$$

A polygonal ray is an infinite collection of points $P_{-7}, P_{-3}, P_{+1}, \ldots$, with indices congruent to $1 \bmod 4$, normalized so that

$$
P_{-7}=(0,0,1), \quad P_{-3}=(1,0,1), \quad P_{+1}=(1,1,1), \quad P_{+5}=(0,1,1) .
$$

These points determine the lines

$$
L_{-5+k}=P_{-7+k} P_{-3+k},
$$

and also the flags

$$
F_{-6+k}=\left(P_{-7+k}, L_{-5+k}\right), \quad F_{-4+k}=\left(P_{-3+k}, L_{-5+k}\right)
$$

We associate corner invariants to the flags, as follows.

$$
\begin{aligned}
& c\left(F_{0+k}\right)=\left[P_{-7+k}, P_{-3+k}, L_{-5+k} L_{3+k}, L_{-5+k} L_{7+k}\right], \\
& c\left(F_{2+k}\right)=\left[P_{9+k}, P_{5+k}, L_{7+k} L_{-1+k}, L_{7+k} L_{-5+k}\right],
\end{aligned}
$$

All these equations are meant for $k=0,4,8,12, \ldots$ Finally, we define

$$
x_{k}=c\left(F_{2 k}\right) ; \quad k=0,1,2,3 \ldots
$$

The quantities $x_{0}, x_{1}, x_{2}, \ldots$ are known as the corner invariants of the ray.

Remark: From the exposition here, it would seem more natural to call these invariants flag invariants, though in the past we have called them corner invariants. 
We would like to go in the other direction. Given a list $\left(x_{0}, x_{1}, x_{2}, \ldots\right)$, we seek a polygonal ray which has this list as its flag invariants. Taking [41], Equation (20), and applying a suitable projective duality, we get the reconstruction formula

$$
P_{9+2 k}=\left[\begin{array}{ccc}
1 & -1 & x_{0} x_{1} \\
1 & 0 & 0 \\
1 & 0 & x_{0} x_{1}
\end{array}\right]\left[\begin{array}{c}
O_{-1}^{3+k} \\
O_{+1}^{3+k} \\
O_{+3}^{3+k}
\end{array}\right] \quad k=0,2,4, \ldots
$$

Multiplying through by the matrix $M^{-1}$, where $M$ is the matrix in Equation (7.31), we get an alternate normalization. Setting

$$
Q_{-7}=\left[\begin{array}{c}
0 \\
x_{0} x_{1} \\
1
\end{array}\right], \quad Q_{-3}=\left[\begin{array}{c}
0 \\
0 \\
x_{0} x_{1}
\end{array}\right], \quad Q_{1}=\left[\begin{array}{l}
1 \\
0 \\
0
\end{array}\right], \quad Q_{5}=\left[\begin{array}{l}
1 \\
1 \\
0
\end{array}\right],
$$

we have

$$
Q_{9+2 k}=\left[\begin{array}{c}
O_{-1}^{3+k} \\
O_{+1}^{3+k} \\
O_{+3}^{3+k}
\end{array}\right] \quad k=0,2,4, \ldots
$$

In case we have a closed $n$-gon, we have

$$
\left[\begin{array}{l}
0 \\
0 \\
1
\end{array}\right]=\left[Q_{-3}\right]=\left[Q_{4 n-3}\right]=\left[Q_{9+2(2 n-6)}\right]=\left[\begin{array}{c}
O_{-1}^{2 n-3} \\
O_{+1}^{2 n-3} \\
O_{+3}^{2 n-3}
\end{array}\right] .
$$

Here, $[\cdot]$ denotes the equivalence class in the projective plane. Equation (7.32) yields $O_{-1}^{2 n-3}=O_{+1}^{2 n-3}=0$. Shifting the vertex labels of our polygon by 1 unit has the effect of shifting the flag invariants by 2 units. Doing all cyclic shifts, we get

$$
O_{a}^{b}=0 \quad b-a=2 n-4,2 n-2, \quad a, b \text { odd. }
$$

Given a polygon $P$ with flag invariants $x_{1}, x_{2}, \ldots$, we consider the dual polygon $P^{*}$. The polygon $P^{*}$ is such that a projective duality carries the lines extending the edges of $P^{*}$ to the points of $P$, and vice versa. When suitably labeled, the flag invariants of $P^{*}$ are $x_{2}, x_{3}, \ldots$ For this reason, Equation (7.33) also holds when both $a$ and $b$ are even. In particular, we have the following $2 n$ relations:

$$
O_{a}^{b}=0, \quad b-a=2 n-4 .
$$

Equation (7.34) tells us that $O_{-1}^{2 n-5}=0$. But now our basic recurrence relation gives

$$
x_{2 n-7} x_{2 n-8} x_{2 n-9} O_{-1}^{2 n-11}-x_{2 n-7} O_{-1}^{2 n-9}+O_{-1}^{2 n-7}=0 .
$$


Note that $x_{0}$ does not occur in this equation. Solving for $x_{2 n-7}$, we get

$$
x_{2 n-7}=R_{n}\left(x_{1}, \ldots, x_{2 n-8}\right),
$$

where $R_{n}$ is the expression that occurs in the map $\Phi$ above. Thanks to Equation (7.34), these equations hold when we shift the indices cyclically by any amount. Thus

$$
x_{2 n-7+k}=R_{n}\left(x_{k+1}, \ldots, x_{2 n-8+k}\right), \quad k=1, \ldots, 2 n .
$$

This is an explanation of why $\Phi^{2 n}$ is the identity.

\section{Relation between the spaces $\mathcal{E}_{w+1, n}, \mathcal{F}_{w+1, n}$, and $\mathcal{C}_{k+1, n}$}

In this section, we give more details about the relations between the main spaces studied in this paper, and complete the proof of Theorem 3.4.1.

8.1. Proof of Theorem 3.4.1, Part (i). Let us prove that the map $\mathcal{E}_{k+1, n} \longrightarrow$ $\mathcal{F}_{k+1, n}$ constructed in Section 3.4 is indeed an isomorphism of algebraic varieties.

A. Let us first prove that this map is a bijection. By the (anti)periodicity assumption (2.2), after $n-k-2$ numbers on each North-East diagonal, there appears 1 , followed by $k$ os. Thus we indeed obtain an array of numbers bounded by a row of $1 \mathrm{~s}$ and $k$ rows of $0 \mathrm{~s}$.

Consider the determinants $D_{i, j}$ defined by (2.4) for $j \geqslant i-1$. We show that $D_{i, j}=1$ by induction on $j$, assuming that $i$ is fixed. By construction, $k+1$ consecutive North-East diagonals of the frieze give a sequence $\left(V_{j}\right)_{j \in \mathbb{Z}}$ of vectors in $\mathbb{R}^{k+1}$ satisfying the difference equation (2.1), where

$$
V_{j}=\left(\begin{array}{c}
d_{i, j} \\
d_{i+1, j} \\
\vdots \\
d_{i+k, j}
\end{array}\right) .
$$

One has $D_{i, j}=\left|V_{j}, V_{j+1}, \ldots, V_{j+k}\right|$. Now we compute the first determinant,

$$
D_{i, i-1}=\left|\begin{array}{cccc}
1 & d_{i, i} & \ldots & d_{i, i+k-1} \\
0 & 1 & \ldots & d_{i+1, i+k-1} \\
\ldots & \ldots & & \ldots \\
0 & 0 & \ldots & 1
\end{array}\right|=1 .
$$

Since the last coefficient in Equation (2.1) satisfied by the sequence of the vectors $\left(V_{j}\right)$ is $a_{i}^{k+1}=(-1)^{k}$, one easily sees that $D_{i, j}=D_{i, j+1}$. Therefore $D_{i, j+1}=$ $D_{i, j}=\cdots=1$. We have proved that the array $d_{i, j}$ is an $\mathrm{SL}_{k+1}$-frieze pattern. 
LEMMA 8.1.1. The defined frieze pattern is tame.

Proof. The space of solutions of the difference equation (2.1) is $k+1$ dimensional; therefore every $(k+2) \times(k+2)$-block cut from $k+2$ consecutive diagonals gives a sequence of linearly dependent vectors. Hence every $(k+2) \times(k+2)$-determinant vanishes.

Conversely, consider a tame $\mathrm{SL}_{k+1}$-frieze pattern. Let $\eta_{j}=\left(\ldots, d_{i, j}, d_{i+1, j}\right.$, ...) be the $j$ th South-East diagonal. We claim that, for every $j$, the diagonal $\eta_{j}$ is a linear combination of $\eta_{j-1}, \ldots, \eta_{j-k-1}$ :

$$
\eta_{j}=a_{j}^{1} \eta_{j-1}-a_{j}^{2} \eta_{j-2}+\cdots+(-1)^{k-1} a_{j}^{k} \eta_{j-k}+(-1)^{k} a_{j}^{k+1} \eta_{j-k-1} .
$$

Indeed, consider a $(k+2) \times(k+2)$-determinant whose last column is on the diagonal $\eta_{j}$. Since the determinant vanishes, the last column is a linear combination of the previous $k+1$ columns. To extend this linear relation to the whole diagonal, slide the $(k+2) \times(k+2)$-determinant in the $\eta$-direction; this yields (8.35).

Next, we claim that $a_{j}^{k+1}=1$. To see this, choose a $(k+1) \times(k+1)$-determinant whose last column is on the diagonal $\eta_{j}$. By the definition of an $\mathrm{SL}_{k+1}$-frieze pattern, this determinant equals 1 . On the other hand, due to relation (8.35), this determinant equals $a_{j}^{k+1}$ times a similar $(k+1) \times(k+1)$-determinant whose last column is on the diagonal $\eta_{j-1}$. The latter also equals 1 ; therefore $a_{j}^{k+1}=1$.

We have shown that each North-East diagonal of the $\mathrm{SL}_{k+1}$-frieze pattern consists of solutions of the linear difference equation (8.35) with $a_{j}^{k+1}=1$, that is, the difference equation (2.1). By the definition of an $\mathrm{SL}_{k+1}$-frieze pattern, these solutions are (anti)periodic. Hence the coefficients are periodic as well.

We proved that the map $\mathcal{E}_{k+1, n} \rightarrow \mathcal{F}_{k+1, n}$ constructed in Section 3.4 is one-toone.

B. Let us now show that this map is a morphism of algebraic varieties defined in Sections 3.1 and 3.2.

Recall that the structure of algebraic variety on $\mathcal{E}_{k+1, n}$ is defined by polynomial equations on the coefficients resulting from the (anti)periodicity. More precisely, these relations can be written in the form

$$
d_{i, i+w}=1, \quad d_{i, j}=0, \quad w<j<n,
$$

where $d_{i, j}$ are defined by (3.9) and calculated according to formulas (5.20) and (5.21) that also make sense for $j \geqslant w$. These polynomial equations guarantee that the solutions of Equation (2.1) are $n$-(anti)periodic. In other words, if $M$ is the monodromy operator of the equation (which is, as is well known, an element of the group $\mathrm{SL}_{k+1}$ ), then the (anti)periodicity condition means that $M=(-1)^{k} \mathrm{Id}$. 
Note that exactly $k(k+2)$ of these equations are algebraically independent, since this is the dimension of $\mathrm{SL}_{k+1}$.

The structure of algebraic variety on the space $\mathcal{F}_{k+1, n}$ is given by the embedding into $\mathrm{Gr}_{k+1, n}$. The Grassmannian itself is an algebraic variety defined by the Plücker relations, and the embedding $\mathcal{F}_{k+1, n} \subset \mathrm{Gr}_{k+1, n}$ is defined by the conditions that some of the Plücker coordinates are equal to each other.

We claim that the map $\mathcal{E}_{k+1, n} \rightarrow \mathcal{F}_{k+1, n}$, constructed in Section 3.4, is a morphism of algebraic varieties (that is, a birational map). Indeed, the coefficients $a_{i}^{j}$ of Equation (2.1) are pull-backs of rational functions in Plücker coordinates; see formula (5.23). Moreover, all the determinants in these formulas are equal to 1 when restricted to $\mathcal{E}_{k+1, n}$. Conversely, the Plücker coordinates restricted to $\mathcal{F}_{k+1, n}$ are polynomial functions in $a_{i}^{j}$. This follows from formulas (5.20) and (5.21), and from the fact that the Plücker coordinates are polynomial in $d_{i, j}$.

This completes the proof of Theorem 3.4.1, Part (i).

8.2. Second isomorphism, when $n$ and $k+1$ are coprime. Let us prove that the spaces of difference equations (2.1) with (anti)periodic solutions and the moduli space of $n$-gons in $\mathbb{R} \mathbb{P}^{k}$ are isomorphic algebraic varieties, provided that the period $n$ and the dimension $k+1$ have no common divisors.

A. We need to check that the map (3.11) is, indeed, a one-to-one correspondence between $\mathcal{E}_{k+1, n}$ and $\mathcal{C}_{k+1, n}$. Let us construct the inverse map to (3.11). Consider a nondegenerate $n$-gon $\left(v_{i}\right)$ in $\mathbb{R P}^{k}$. Choose an arbitrary lift $\left(\tilde{V}_{i}\right) \in \mathbb{R}^{k+1}$ of the vertices. The $k+1$ coordinates $\tilde{V}_{i}^{(1)}, \ldots, \tilde{V}_{i}^{(k+1)}$ of the vertices of this $n$-gon are solutions to some (and the same) difference equation (2.1) if and only if the determinant (3.10) is constant (that is, independent of $i$ ). We thus wish to define a new lift $V_{i}=t_{i} \tilde{V}_{i}$ such that

$$
t_{i} t_{i+1} \cdots t_{i+k}\left|\tilde{V}_{i}, \tilde{V}_{i+1}, \ldots, \tilde{V}_{i+k}\right|=1 \text {. }
$$

This system of equations on $t_{1}, \ldots, t_{n}$ has a unique solution if and only if $n$ and $k+1$ are coprime. Finally, two projectively equivalent $n$-gons correspond to the same equation. Thus the map (3.11) is a bijection.

B. The structures of algebraic varieties are in full accordance, since the projection from $\mathrm{Gr}_{k+1, n}$ to $\mathcal{C}_{k+1, n}$ is given by the projection with respect to the $\mathbb{T}^{n-1}$-action which is an algebraic action of an algebraic group.

Theorem 3.4.1, Part (ii) is proved.

8.3. Proof of Proposition 3.4.4. Consider finally the case where $n$ and $k+1$ have common divisors. Suppose that $\operatorname{gcd}(n, k+1)=q \neq 1$. In this case, the constructed map is not injective, and its image is a subvariety in the moduli space of polygons. 
As before, we assign an $n$-gon $V_{1}, \ldots, V_{n} \in \mathbb{R}^{k+1}$ to a difference equation. Given numbers $t_{0}, \ldots, t_{q-1}$ whose product is 1 , we can rescale

$$
V_{j} \mapsto t_{j \bmod q} V_{j}, \quad j=1, \ldots, n,
$$

keeping the determinants $\left|V_{i}, V_{i+1}, \ldots, V_{i+k}\right|$ intact. This action of $\left(\mathbb{R}^{*}\right)^{q-1}$ does not affect the projection of the polygons to $\mathbb{R} \mathbb{P}^{k}$. Thus the fiber of this projection is at least $q-1$-dimensional.

To find the dimension of the fiber, we need to consider the system of equations

$$
t_{i} t_{i+1} \cdots t_{i+k}=1, \quad i=1, \ldots, n,
$$

where, as usual, the indices are understood cyclically $\bmod n$. Taking logarithms, this is equivalent to a linear system with the circulant matrix

$$
\left(\begin{array}{ccccccccc}
1 & 1 & \ldots & 1 & 1 & 0 & \ldots & 0 & 0 \\
0 & 1 & \ldots & 1 & 1 & 1 & 0 & \ldots & 0 \\
& & \ddots & & & & \ddots & & \\
0 & 0 & \ldots & 0 & 1 & 1 & 1 & \ldots & 1 \\
1 & 0 & \ldots & 0 & 0 & 1 & 1 & \ldots & 1 \\
& & \ddots & & & & \ddots & & \\
1 & 1 & \ldots & 1 & 0 & 0 & \ldots & 0 & 1
\end{array}\right)
$$

with $k+11$ s in each row and column.

The eigenvalues of such a matrix are given by the formula

$$
1+\omega_{j}+\omega_{j}^{2}+\cdots+\omega_{j}^{k}, \quad j=0,1, \ldots, n-1,
$$

where $\omega_{j}=\exp (2 \pi \mathbf{i} j / n)$ is $n$th root of 1 ; see [10]. If $j>0$, the latter sum equals

$$
\frac{\omega_{j}^{k+1}-1}{\omega_{j}-1},
$$

and it equals 0 if and only if $j(k+1)=0 \bmod n$. This equation has $q-1$ solutions; hence the circulant matrix has corank $q-1$. Proposition 3.4.4 is proved.

8.4. The $\mathrm{SL}_{2}$-case: relations to Teichmüller theory. Now, we give a more geometric description of the image of the map (3.11) in the case when $k=1$. If $n$ is odd then this map is one-to-one, but if $n$ is even then its image has 
codimension 1 . To describe this image, we need some basic facts from decorated Teichmüller theory [36, 37].

Consider $\mathbb{R} \mathbb{P}^{1}$ as the circle at infinity of the hyperbolic plane. Then a polygon in $\mathbb{R} \mathbb{P}^{1}$ can be thought of as an ideal polygon in the hyperbolic plane $\mathcal{H}^{2}$. A decoration of an ideal $n$-gon is a choice of horocycles centered at its vertices.

Choose a decoration, and define the side length of the polygon as the signed hyperbolic distance between the intersection points of the respective horocycles with this side; the convention is that if the two consecutive horocycles are disjoint then the respective distance is positive (one can always assume that the horocycles are 'small' enough). Denoting the side length by $\delta$, the lambda length is defined as $\lambda=\exp (\delta / 2)$.

Let $n$ be even. Define the alternating perimeter length of an ideal $n$-gon: choose a decoration and consider the alternating sum of the side lengths. The alternating perimeter length of an ideal even-gon does not depend on the decoration: changing a horocycle adds (or subtracts) the same length to two adjacent sides of the polygon, and does not change the alternating sum.

PROPOSITION 8.4.1. The image of the map (3.11) with $k=1$ and $n$ even consists of polygons with zero alternating perimeter length.

Proof. Let $\left(v_{i}\right)$ be a polygon in $\mathbb{R P}^{1}$. Let $x_{i}=\left[v_{i-1}, v_{i}, v_{i+1}, v_{i+2}\right]$ be the crossratio of the four consecutive vertices. Of six possible definitions of cross-ratio, we use the following one:

$$
\left[t_{1}, t_{2}, t_{3}, t_{4}\right]=\frac{\left(t_{1}-t_{3}\right)\left(t_{2}-t_{4}\right)}{\left(t_{1}-t_{2}\right)\left(t_{3}-t_{4}\right)} .
$$

This is the reciprocal of the formula in Equation (7.30).

We claim that a $2 n$-gon is in the image of (3.11) if and only if

$$
\prod_{i \text { odd }} x_{i}=\prod_{i \text { even }} x_{i}
$$

Indeed, let $\left(V_{i}\right) \subset \mathbb{R}^{2}$ be an antiperiodic solution to the discrete Hill's equation

$$
V_{i+1}=c_{i} V_{i}-V_{i-1}
$$

with $\left|V_{i}, V_{i+1}\right|=1$. Then

$$
x_{i}=\frac{\left|V_{i-1}, V_{i+1}\right|\left|V_{i}, V_{i+2}\right|}{\left|V_{i-1}, V_{i}\right|\left|V_{i+1}, V_{i+2}\right|}=c_{i} c_{i+1} .
$$

Therefore (8.36) holds. 
Conversely, let (8.36) hold. Let $\left(\tilde{V}_{i}\right)$ be a lift of $\left(v_{i}\right)$ to $\mathbb{R}^{2}$ with $\left|\tilde{V}_{i}, \tilde{V}_{i+1}\right|>0$. As before, we want to renormalize these vectors so that the consecutive determinants equal 1 . This boils down to solving the system of equations $t_{i} t_{i+1}\left|\tilde{V}_{i}, \tilde{V}_{i+1}\right|=1$. This system has a solution if and only if

$$
\prod_{i \text { odd }}\left|\tilde{V}_{i}, \tilde{V}_{i+1}\right|=\prod_{i \text { even }}\left|\tilde{V}_{i}, \tilde{V}_{i+1}\right| \cdot
$$

On the other hand, one computes that

$$
1=\frac{\prod_{i \text { odd }} x_{i}}{\prod_{i \text { even }} x_{i}}=\left(\frac{\prod_{i \text { even }}\left|\tilde{V}_{i}, \tilde{V}_{i+1}\right|}{\prod_{i \text { odd }}\left|\tilde{V}_{i}, \tilde{V}_{i+1}\right|}\right)^{2},
$$

and the desired rescaling exists.

Finally, one relates cross-ratios with lambda lengths; see [36, 37]:

$$
\left[v_{i-1}, v_{i}, v_{i+1}, v_{i+2}\right]=\frac{\lambda_{i-1, i+1} \lambda_{1, i+2}}{\lambda_{i-1, i} \lambda_{i+1, i+2}} .
$$

Therefore

$$
1=\frac{\prod_{i \text { odd }} x_{i}}{\prod_{i \text { even }} x_{i}}=\left(\frac{\prod_{i \text { even }} \lambda_{i, i+1}}{\prod_{i \text { odd }} \lambda_{i, i+1}}\right)^{2}=e^{\sum(-1)^{i} \delta_{i}} .
$$

It follows that the alternating perimeter length is zero.

\section{Acknowledgements}

We are pleased to thank D. Leites and A. Veselov for enlightening discussions. This project originated at the Institut Mathématique de Jussieu (IMJ), Université Paris 6. S.T. is grateful to IMJ for its hospitality. S.M.-G. and V.O. were partially supported by the PICS05974 'PENTAFRIZ' of CNRS. R.E.S. was supported by NSF grant DMS-1204471. S.T. was supported by NSF grant DMS-1105442.

\section{References}

[1] D. André, 'Terme général d'un série quelconque déterminée à la façon des séries récurrentes', Ann. Sci. Éc. Norm. Super. 7 (2) (1878), 375-408.

[2] I. Assem, C. Reutenauer and D. Smith, 'Friezes', Adv. Math. 225 (2010), 3134-3165.

[3] A. Berenstein, S. Fomin and A. Zelevinsky, 'Parametrizations of canonical bases and totally positive matrices', Adv. Math. 122 (1996), 49-149.

[4] F. Bergeron and C. Reutenauer, ' $\mathrm{SL}_{k}$-tilings of the plane', Illinois J. Math. 54 (2010), 263-300. 
[5] P. Caldero and F. Chapoton, 'Cluster algebras as Hall algebras of quiver representations', Comment. Math. Helv. 81 (2006), 595-616.

[6] A. B. Coble, 'Associated sets of points', Trans. Amer. Math. Soc. 24 (1922), 1-20.

[7] A. B. Coble, Algebraic Geometry and Theta Functions, vol. X. (AMS, Providence, RI, 1961).

[8] J. H. Conway and H. S. M. Coxeter, 'Triangulated polygons and frieze patterns', Math. Gaz. 57 (1973), 87-94 and 175-183.

[9] H. S. M. Coxeter, 'Frieze patterns', Acta Arith. 18 (1971), 297-310.

[10] Ph. Davis, Circulant Matrices, (John Wiley \& Sons, New York-Chichester-Brisbane, 1979).

[11] P. Di Francesco and R. Kedem, 'T-systems with boundaries from network solutions', Electron. J. Combin. 20 (1) (2013), 62.

[12] I. Dolgachev and D. Ortland, 'Points sets in projective spaces and theta functions', Astérisque 165 (1988).

[13] B. Dubrovin, A. Fomenko and S. Novikov, 'Modern geometry-methods and applications. Part I', in The Geometry of Surfaces, Transformation Groups, and Fields, 2nd edn, (SpringerVerlag, New York, 1992).

[14] D. Eisenbud and S. Popescu, 'The projective geometry of the Gale transform', J. Algebra 220 (2000), 127-173.

[15] D. Fuchs and S. Tabachnikov, 'Self-dual polygons and self-dual curves', Funct. Anal. Other Math. 2 (2009), 203-220.

[16] W. Fulton, Young tableaux, with applications to representation theory and geometry, London Mathematical Society Student Texts 35 (Cambridge University Press, Cambridge, 1997).

[17] D. Gale, 'Neighboring vertices on a convex polyhedron', in Linear Inequalities and Related Systems, (eds H. W. Kuhn and A. W. Tucker), Annals of Math Studies 38 (Princeton University Press, Princeton, NJ, 1956), 255-263.

[18] C. F. Gauss, 'Pentagramma Mirificum', Werke, Bd. III, 481-490; Bd VIII 106-111.

[19] M. Gekhtman, M. Shapiro, S. Tabachnikov and A. Vainshtein, 'Higher pentagram maps, weighted directed networks, and cluster dynamics', Electron. Res. Announc. Math. Sci. 19 (2012), 1-17.

[20] M. Gekhtman, M. Shapiro, S. Tabachnikov and A. Vainshtein, 'Integrable cluster dynamics of directed networks and pentagram maps', arXiv:14061883.

[21] I. M. Gelfand and R. D. MacPherson, 'Geometry in Grassmannians and a generalization of the dilogarithm', Adv. Math. 44 (1982), 279-312.

[22] C. Jordan, Calculus of Finite Differences, 3rd edn, (Chelsea Publishing Co, New York, 1965).

[23] M. Kapranov, 'Chow quotients of Grassmannians, I', in I. M. Gel'fand Seminar, Advances in Soviet Math. 16 Part 2 (Amer. Math. Soc., Providence, RI, 1993), 29-110.

[24] B. Keller, 'The periodicity conjecture for pairs of Dynkin diagrams', Ann. of Math. (2) 177 (2013), 111-170.

[25] B. Khesin and F. Soloviev, 'Integrability of a space pentagram map', Math. Ann. 357 (2013), 1005-1047.

[26] B. Khesin and F. Soloviev, 'The geometry of dented pentagram maps', J. Eur. Math. Soc. (to appear).

[27] I. Krichever, 'Commuting difference operators and the combinatorial Gale transform', arXiv: 14034629.

[28] I. Krichever and S. Novikov, 'A two-dimensionalized Toda chain, commuting difference operators, and holomorphic vector bundles', Russian Math. Surveys 58 (2003), 473-510. 
[29] G. Mari-Beffa, 'On generalizations of the pentagram map: discretizations of AGD flows', J. Nonlinear Sci. 23 (2013), 303-334.

[30] G. Mari-Beffa, 'On integrable generalizations of the pentagram map', IMRN (to appear).

[31] S. Morier-Genoud, 'Arithmetics of 2-friezes', J. Algebraic Combin. 36 (2012), 515-539.

[32] S. Morier-Genoud, V. Ovsienko and S. Tabachnikov, '2-Frieze patterns and the cluster structure of the space of polygons', Ann. Inst. Fourier 62 (2012), 937-987.

[33] V. Ovsienko and S. Tabachnikov, Projective Differential Geometry Old and New: From the Schwarzian Derivative to the Cohomology of Diffeomorphism Groups, (Cambridge University Press, Cambridge, 2005).

[34] V. Ovsienko, R. Schwartz and S. Tabachnikov, 'The pentagram map: a discrete integrable system', Comm. Math. Phys. 299 (2) (2010), 409-446.

[35] V. Ovsienko, R. Schwartz and S. Tabachnikov, 'Liouville-Arnold integrability of the pentagram map on closed polygons', Duke Math. J. 162 (2013), 2149-2196.

[36] R. Penner, Decorated Teichmüller Theory, (European Mathematical Society, 2012).

[37] R. Penner, 'Lambda lengths'. http://wwwctqmaudk/research/MCS/lambdalengthspdf.

[38] J. Propp, 'The combinatorics of frieze patterns and Markoff numbers', arXiv:math/0511633.

[39] V. Schechtman, 'Pentagramma Mirificum and elliptic functions', arXiv:11063633.

[40] R. Schwartz, 'The pentagram map', Experiment. Math. 1 (1992), 71-81.

[41] R. Schwartz, 'Discrete monodromy, pentagrams, and the method of condensation', J. Fixed Point Theory Appl. 3 (2008), 379-409.

[42] R. Schwartz, 'Pentagram spirals', Experiment. Math. 22 (2013), 384-405.

[43] F. Soloviev, 'Integrability of the Pentagram map', Duke Math. J. 162 (2013), 2815-2853.

[44] T. A. Springer, Linear algebraic groups, Progress in Mathematics, vol. 9. (Birkhäuser Boston, Inc, Boston, MA, 1998).

[45] A. Y. Volkov, 'On the periodicity conjecture for $Y$-systems', Comm. Math. Phys. 276 (2007), 509-517.

[46] A. Zabrodin, 'Discrete Hirotaś equation in quantum integrable models', Internat. J. Modern Phys. B 11 (1997), 3125-3158. 